\title{
Intellectual Delinquencies Among Youth and Ways of Prevention from the Perspective of Youth and Social Workers
}

\author{
Jameelah M. Allaboon \\ Associate Professor of Social Work, College of Social Work, \\ Princess Nourah bint Abdulrahman University, \\ Airport Road, King Khalid International Airport, \\ Riyadh 11564, Saudi Arabia
}

DOI: https://doi.org/10.36941/jesr-2022-0037

\section{Abstract}

The present research paper aimed to identify intellectual delinquencies and their factors among youth and suggest ways of prevention from the perspective of youth and social workers. Therefore, it adopted the descriptive analytical approach and applied a questionnaire to a sample of (209) students aged (18-30) years at some Saudi universities and (90) social workers. The results showed the highest intellectual delinquencies among youth from the perspective of youth and social workers, the (family, societal, personal, and educational) factors, and the suggested ways of prevention. The study recommended establishing a special unit at the university to provide training courses to students to develop their skills and raise their awareness about current issues, such as intellectual delinquencies.

Keywords: Intellectual delinquencies, Youth, Prevention, Social workers, Social work

\section{Introduction}

Recently, several intellectual delinquencies in thinking, sayings, or actions have resulted in social, political, and religious problems. Therefore, researchers are motivated to investigate those changes and overcome the crisis of the Arab World. Intellectual delinquency is a belief in specific ideas, although they violate the common laws and norms, including the principles of justice, freedom, and equality (Alkhatib 2015). According to (Call 2004), intellectual delinquency denotes the mismatch of personal thinking, impressions, and perceptions with the typical intellectual, political, or cultural values and principles.

To interpret intellectual delinquency socially, the structural functionalism developed by Parsons believes that people acquire the fundamental values, standards, and roles through socialization. Still, failure to achieve or accept them indicates delinquency (Abdelgawad 2009). Alsharary (Alsharary 2007) argues that intellectual delinquency, as a social phenomenon, signifies the social context because it results from the lack of relationship with social groups that manage and guide behavior or the anomie among some social groups lacking social guidance and discipline. Therefore, the present research paper stresses highlighting and reformulating the constituents of the cultural structure of university students with intellectual delinquency through guided programs to handle the problems and manifestations of intellectual delinquency.

Ronald (Ronald 2005) believes that intellectual delinquency among the youth is driven by several psychological, social, political, and economic reasons. Alhokeel (Alhokeel 2013) concludes that the bad company, negative impact of misleading media, websites with deviant content, lack of 
prevention programs, and poor religious restraints cause intellectual delinquency. Al-Khataibeh et al. (Al-Khataibeh et al. 2014) illustrate that social reasons are ranked first in intellectual delinquency among youth, followed by the political, academic, and economic ones. In contrast, Alaly and KhairBek (Alaly and Khair-Bek 2017) argue that family breakdown and poverty are the leading factors of intellectual delinquency.

Intellectual delinquency is manifested by the tendency to isolation, aggression with peers, and fanaticism (Al-tayyar 2017). It has several negative impacts, including intellectual imbalances, insecure ideas and beliefs, aggressive speech and action, corrupt values, terrorism, lack of security, and weak family ties (Naser 2007). It endangers individuals because it changes the principles, values, ideas, and believes into negative ones that affect social behavior (Sonbol 2013).

Intellectual delinquency is highly dangerous among the youth because they are the essential pillar of society and tend to imitate and mimic (Larton 2005). Abu-Arrad (Abu-Arrad 2010) shows high violence among university students, which affects their national and religious identity. Additionally, Alkhathlan (Alkhathlan 2017) reports that intellectual delinquency is high among university youth and threatens family and society.

Aldahmash (Aldahmash 2019) illustrates the valuable efforts done by the Saudi Ministry of Education following Saudi Vision 2030 to achieve intellectual security in curricula, promoting activities and events, or qualifying teachers. He adds that Saudi Arabia has made many efforts to handle intellectual delinquency, such as the conference of "Terrorism between the Extremism of Thought and the Ideology of Extremism" in 2011 at the Islamic University of Medinah, the symposium of "Electronic Terrorism: Dangers and Prevention" in 2014 at Imam Mohammad Ibn Saud Islamic University, the international conference of "the Role of Arab Media in Terrorism Confrontation" in 2015 at Naif Arab University for Security Sciences, and the symposium of "University Responsibility in Promoting Preventive Aspects to Confront Terrorist Organizations" in 2017 at Imam Mohammad Ibn Saud Islamic University. However, intellectual delinquency spreads.

The preventive role against intellectual delinquency is vital, as shown in plans, programs, and curricula, to raise students soundly, equip them with religious and moral principles and values, develop their feeling of belonging and loyalty, and qualify them to adhere to moderation and social responsibility (Aljahny 2011). Alnaeemy (Alnaeemy 2016) concludes that confronting intellectual delinquency requires the efforts of all stakeholders.

Several studies addressed the prevention of intellectual delinquency. For instance, Dillon et al. (Dillon et al. 2008) reported that good parental monitoring keeps children from intellectual delinquency. Clinch (Clinch 2011) stressed confronting intellectual delinquency and promoting belonging to the school, society, and nation among school students in England based on their perceptions of intellectual prevention programs. To prevent intellectual delinquency among students, Alhokeel (Alhokeel 2013) suggested the interest in guidance and psychological counseling programs, adopting the culture of dialog, developing prevention programs for students with delinquency and parents to define the ways of prevention before emergence, and publishing the legal opinion on intellectual delinquency in visible locations in the educational institution. Alamry (Alamry 2014) argued that a course on (security culture) should be delivered in schools and universities to cover the values and issues of the security culture.

The research problem is defined in the prevalence of intellectual delinquency among the students of Arab and Islamic societies, including Saudi Arabia, because of the lack of knowledge of this group, turning them into a method of destruction and disturbance. Therefore, the best solution is to address the causes of intellectual delinquency scientifically. The present paper tries to answer the following major question:

What are the intellectual delinquencies among youth and ways of prevention from the perspective of university students and social workers?

It is further divided into the following minor questions:

1. What are the intellectual delinquencies among youth from the perspective of university students and social workers? 
2. What are the factors of intellectual delinquencies among youth from the perspective of university students and social workers?

3. What are the suggested ways of preventing intellectual delinquencies among youth from the perspective of university students and social workers?

\section{Methodology}

\subsection{Method}

The present study is analytical descriptive. The population covers university students and faculty of social work. It aims to identify the intellectual delinquencies, factors, and ways of prevention among youth. The author adopted the social survey approach of university students and social workers because it is the best to the nature of the study as it helps describe and analyze the data to conclude and generalize findings.

\subsection{Population}

The population includes all students at Saudi universities and faculty of social work in the academic year 2018/2019.

\subsection{Sampling}

The sample comprises (209) students aged (18-30) years at some Saudi universities and (90) social workers, as shown in tables (1) and (2).

Table 1: Describing the sample (university students)

\begin{tabular}{|c|c|c|c|}
\hline \multicolumn{2}{|l|}{ variables } & Freq. & $\%$ \\
\hline \multirow[t]{2}{*}{ Gender } & Female & 144 & 68.9 \\
\hline & Male & 65 & 31.1 \\
\hline \multirow[t]{4}{*}{ Age } & 18 to less than 22 years & 8 & 3.8 \\
\hline & 22 to less than 26 years & 80 & 38.3 \\
\hline & 26 to less than 30 years & 98 & 46.9 \\
\hline & 30 years and more & 23 & 11.0 \\
\hline \multirow[t]{11}{*}{ University } & Princess Nourah Bint Abdulrahman University & 85 & 40.6 \\
\hline & Imam Abdulrahman bin Faisal University & 16 & 7.7 \\
\hline & King Saud University & 17 & 8.1 \\
\hline & King Abdulaziz University & 1 & 5. \\
\hline & Majmaah University & 2 & 1.0 \\
\hline & Prince Sattam Bin Abdulaziz University & 4 & 1.9 \\
\hline & Mzahmia University & 2 & 1.0 \\
\hline & King Faisal University & 4 & 1.9 \\
\hline & King Khalid University & 3 & 1.4 \\
\hline & Almaarefa University & 4 & 1.9 \\
\hline & Other & 71 & 34.0 \\
\hline \multicolumn{2}{|l|}{ Total } & 209 & $\% 100$ \\
\hline
\end{tabular}

Table (1) shows the difference in the samples in terms of gender, age, and university.

Table 2: Describing the sample (social workers)

\begin{tabular}{|l|l|c|c|}
\hline Variables & Female & Freq. & \% \\
\cline { 2 - 4 } Gender & Male & 59 & 65.6 \\
\hline \multirow{4}{*}{ Academic degree } & Professor & 31 & 34.4 \\
\cline { 2 - 4 } & Assistant Professor & 18 & 20.0 \\
\cline { 2 - 4 } & Associate Professor & 28 & 31.2 \\
\cline { 2 - 4 } & Lecturer & 24 & 26.6 \\
\hline \multicolumn{2}{|l|}{ Total } & 20 & 22.2 \\
\hline
\end{tabular}


Table (2) shows the difference in the samples in terms of gender and academic degree.

\subsection{Tools}

\subsubsection{The questionnaire}

The questionnaire aimed to identify the intellectual delinquencies and factors among youth and the suggested ways of prevention from the perspective of university students and social workers. After reviewing the literature and interviewing specialists on the domains and phrasing the items, the first form of the questionnaire was prepared and included (19) items in the first domain, (12) items in the second domain, (12) items in the third domain, (12) items in the fourth domain, (12) items in the fifth domain, (11) items in the sixth domain, (11) items in the seventh domain, (11) items in the eighth domain, and (11) items in the ninth domain. Those items were concise, accurate, and clear.

The final form of the questionnaire included two parts. Part I covered personal data, i.e., gender, age, and university (first questionnaire) and gender and academic degree (second questionnaire). Part II comprised nine domains. The author employed a three-point Likert scale to measure the relevance of each item (agree, undecided, and disagree).

- Reviewers' validity (face validity)

The first form of the questionnaire was submitted to (11) reviewers of the faculty members at Saudi universities to determine the items' content, relevance, phrasing, clarity, and appropriateness. The reviewers suggested some essential modifications, e.g., modifying, replacing, and paraphrasing some items. After making modifications, the final form of the questionnaire was obtained.

- Construct validity

The construct validity between each item and the total degree of the domain was calculated by applying to a pilot sample of (40) items. All correlation coefficients were in the closed interval [0.376o.965], suggesting that all correlation coefficients were statistically significant at the level of o.o1, and the questionnaire was valid.

\section{- Reliability}

The reliability of the questionnaire was estimated using Cronbach's alpha that showed that all reliability coefficients were significant at the level of o.o1 and ranged from 0.8699 to 0.9598 . The total reliability coefficient equaled o.8939, denoting high reliability.

\section{Results and Discussion}

To answer the first question, the frequencies, percentages, arithmetic means, standard deviations, and ranks of the responses to intellectual delinquencies among youth were calculated, as shown in table (3).

Table 3: Responses of the participants to the intellectual delinquencies among youth

\begin{tabular}{|c|c|c|c|c|c|c|c|c|c|}
\hline \multirow[t]{2}{*}{ No. } & \multirow{2}{*}{\multicolumn{2}{|c|}{ Item }} & \multicolumn{4}{|c|}{ Agreement } & \multirow{2}{*}{$\begin{array}{c}\text { Arithmetic } \\
\text { mean }\end{array}$} & \multirow{2}{*}{$\begin{array}{l}\text { Standard } \\
\text { deviation }\end{array}$} & \multirow{2}{*}{ Rank } \\
\hline & & & & Agree & Undecided & Disagree & & & \\
\hline \multirow[t]{6}{*}{7} & \multirow{6}{*}{ Fanaticism } & \multirow[t]{2}{*}{ Workers } & $\mathrm{F}$ & 64 & 20 & 6 & \multirow{2}{*}{2.64} & \multirow{2}{*}{0.605} & \multirow{6}{*}{1} \\
\hline & & & $\%$ & 71.1 & 22.2 & 6.7 & & & \\
\hline & & \multirow[t]{2}{*}{ Students } & $\mathrm{F}$ & 130 & 58 & 21 & \multirow{2}{*}{2.52} & \multirow{2}{*}{0.673} & \\
\hline & & & $\%$ & 62.2 & 27.8 & 10.0 & & & \\
\hline & & \multirow[t]{2}{*}{ Total } & $\mathrm{F}$ & 194 & 78 & 27 & \multirow{2}{*}{2.56} & \multirow{2}{*}{0.665} & \\
\hline & & & $\%$ & 64.9 & 26.1 & 9.0 & & & \\
\hline \multirow[t]{6}{*}{13} & \multirow[t]{6}{*}{ Unbalanced view of issues } & \multirow[t]{2}{*}{ Workers } & $\mathrm{F}$ & 58 & 30 & 2 & \multirow{2}{*}{2.62} & \multirow{2}{*}{0.532} & \multirow{6}{*}{2} \\
\hline & & & $\%$ & 64.4 & $33 \cdot 3$ & 2.2 & & & \\
\hline & & \multirow[t]{2}{*}{ Students } & $\mathrm{F}$ & 104 & 75 & 30 & \multirow{2}{*}{2.35} & \multirow{2}{*}{0.720} & \\
\hline & & & $\%$ & 49.8 & 35.9 & 14.4 & & & \\
\hline & & \multirow[t]{2}{*}{ Total } & $\mathrm{F}$ & 162 & 105 & 32 & \multirow{2}{*}{2.43} & \multirow{2}{*}{0.679} & \\
\hline & & & $\%$ & 54.2 & 35.1 & 10.7 & & & \\
\hline \multirow[t]{2}{*}{10} & \multirow{2}{*}{$\begin{array}{l}\text { Closed-mindedness towards } \\
\text { personal and public problems }\end{array}$} & \multirow[t]{2}{*}{ Workers } & $\mathrm{F}$ & 57 & 28 & 5 & \multirow{2}{*}{2.58} & \multirow{2}{*}{0.599} & \multirow{2}{*}{3} \\
\hline & & & $\%$ & 63.3 & 31.1 & 5.6 & & & \\
\hline
\end{tabular}




\begin{tabular}{|c|c|c|c|c|c|c|c|c|c|}
\hline \multirow[t]{6}{*}{ No. } & \multirow{6}{*}{$\begin{array}{l}\text { Item } \\
\text { and issues }\end{array}$} & & \multicolumn{4}{|c|}{ Agreement } & \multirow{2}{*}{$\begin{array}{c}\text { Arithmetic } \\
\text { mean }\end{array}$} & \multirow{2}{*}{$\begin{array}{l}\text { Standard } \\
\text { deviation }\end{array}$} & \multirow{6}{*}{ Rank } \\
\hline & & & & Agree & Undecided & Disagree & & & \\
\hline & & \multirow[t]{2}{*}{ Students } & $\mathrm{F}$ & 107 & 63 & 39 & \multirow{2}{*}{2.33} & \multirow{2}{*}{0.772} & \\
\hline & & & $\%$ & 51.2 & 30.1 & 18.7 & & & \\
\hline & & \multirow[t]{2}{*}{ Total } & $\mathrm{F}$ & 164 & 91 & 44 & \multirow[b]{2}{*}{2.40} & & \\
\hline & & & $\%$ & 54.8 & 30.4 & 14.7 & & 0.732 & \\
\hline 6 & Unjustified extremism & Workers & $\mathrm{F}$ & 62 & 23 & 5 & 260 & $0-80$ & \\
\hline & & & $\%$ & 68.9 & 25.6 & 5.6 & 2.03 & 0.509 & \\
\hline & & Students & $\mathrm{F}$ & 104 & 62 & 43 & 230 & -788 & \\
\hline & & & $\%$ & 49.8 & 29.7 & 20.6 & 2.29 & 0.700 & 4 \\
\hline & & Total & $\mathrm{F}$ & 166 & 85 & 48 & & & \\
\hline & & & $\%$ & 55.5 & 28.4 & 16.1 & 2.39 & $0.75^{\circ}$ & \\
\hline 1 & Misinformation and deception & Workers & $\mathrm{F}$ & 49 & 33 & 8 & & & \\
\hline & & & $\%$ & 54.4 & 36.7 & 8.9 & 2.46 & 0.656 & \\
\hline & & Students & $\mathrm{F}$ & 77 & 107 & 25 & & & \\
\hline & & & $\%$ & 36.8 & 51.2 & 12.0 & 2.25 & 0.654 & 5 \\
\hline & & Total & $\mathrm{F}$ & 126 & 140 & 33 & & 66 & \\
\hline & & & $\%$ & 42.1 & 46.8 & 11.0 & 2.31 & 0.661 & \\
\hline 8 & Tendency to enmity and revenge & Workers & $\mathrm{F}$ & 49 & 34 & 7 & & 0640 & \\
\hline & & & $\%$ & 54.4 & 37.8 & 7.8 & 2.47 & 0.640 & \\
\hline & & Students & $\mathrm{F}$ & 87 & 83 & 39 & 2,23 & 0772 & 6 \\
\hline & & & $\%$ & 41.6 & 39.7 & 18.7 & 2.23 & 0.143 & 0 \\
\hline & & Total & $\mathrm{F}$ & 136 & 117 & 46 & 230 & 1 & \\
\hline & & & $\%$ & 45.5 & 39.1 & 15.4 & 2.30 & 0.721 & \\
\hline 5 & Distortion of facts & Workers & $\mathrm{F}$ & 53 & 29 & 8 & 250 & 0658 & \\
\hline & & & $\%$ & 58.9 & 32.2 & 8.9 & 2.50 & 0.050 & \\
\hline & & Students & $\mathrm{F}$ & 90 & 72 & 47 & & & \\
\hline & & & $\%$ & 43.1 & 34.4 & 22.5 & 2.21 & 0.785 & 7 \\
\hline & & Total & $\mathrm{F}$ & 143 & 101 & 55 & & 0 760 & \\
\hline & & & $\%$ & 47.8 & 33.8 & 18.4 & 2.29 & 0.760 & \\
\hline 19 & Adhering some socially rejected & Workers & $\mathrm{F}$ & 46 & 33 & 11 & 232 & 0608 & \\
\hline & ideas & & $\%$ & 51.1 & 36.7 & 12.2 & 2.39 & 0.698 & \\
\hline & & Students & $\mathrm{F}$ & 86 & 80 & 43 & 2 & ה & 8 \\
\hline & & & $\%$ & 41.1 & 38.3 & 20.6 & 2.21 & 0.760 & 8 \\
\hline & & Total & $\mathrm{F}$ & 132 & 113 & 54 & 2.26 & 746 & \\
\hline & & & $\%$ & 44.1 & 37.8 & 18.1 & 2.26 & 0.740 & \\
\hline 9 & Rejection of intellectual dialogue & Workers & $\mathrm{F}$ & 46 & 35 & 9 & 24 & 0660 & \\
\hline & with the other & & $\%$ & 51.1 & 38.9 & 10.0 & 2.41 & 0.009 & \\
\hline & & Students & $\mathrm{F}$ & 78 & 87 & 44 & 2,16 & 0778 & \\
\hline & & & $\%$ & $37 \cdot 3$ & 41.6 & 21.1 & 2.16 & 0.748 & 9 \\
\hline & & Total & $\mathrm{F}$ & 124 & 122 & 53 & & & \\
\hline & & & $\%$ & 41.5 & 40.8 & 17.7 & 2.24 & 0.733 & \\
\hline 14 & Adopting and promoting & Workers & $\mathrm{F}$ & 47 & 27 & 16 & & -76 & \\
\hline & destructive ideas & & $\%$ & 52.2 & 30.0 & 17.8 & 2.34 & 0.767 & \\
\hline & & Students & $\mathrm{F}$ & 91 & 60 & 58 & -1 & $\Omega_{-}$ & \\
\hline & & & $\%$ & 43.5 & 28.7 & 27.8 & 2.16 & 0.831 & 10 \\
\hline & & Total & $\mathrm{F}$ & 138 & 87 & 74 & 2 & 8,6 & \\
\hline & & & $\%$ & 46.2 & 29.1 & 24.7 & 2.21 & 0.816 & \\
\hline 12 & Obligating the self and others to & Workers & $\mathrm{F}$ & 40 & 35 & 15 & 2,8 & 0725 & \\
\hline & do unnecessary things & & $\%$ & 44.4 & 38.9 & 16.7 & & 0.735 & \\
\hline & & Students & $\mathrm{F}$ & 83 & 60 & 66 & 208 & 0842 & 11 \\
\hline & & & $\%$ & 39.7 & 28.7 & 31.6 & & & 11 \\
\hline & & Total & $\mathrm{F}$ & 123 & 95 & 81 & 2,4 & $8_{15}$ & \\
\hline & & & $\%$ & 41.1 & 31.8 & 27.1 & 2.14 & 0.015 & \\
\hline 11 & Rejection of innovation and & Workers & $\mathrm{F}$ & 38 & 38 & 14 & & 6 & \\
\hline & diversity & & $\%$ & 42.2 & 42.2 & 15.6 & 2.27 & 0.716 & \\
\hline & & Students & $\mathrm{F}$ & 83 & 58 & 68 & & & \\
\hline & & & $\%$ & 39.7 & 27.8 & 32.5 & 2.07 & 0.849 & 12 \\
\hline & & Total & $\mathrm{F}$ & 121 & 96 & 82 & & $0_{-}$ & \\
\hline & & & $\%$ & 40.5 & 32.1 & 27.4 & 2.13 & 0.815 & \\
\hline 3 & Exaggeration and extremism in & Workers & $\mathrm{F}$ & 40 & 35 & 15 & 2,28 & & \\
\hline & applying Islamic law & & $\%$ & 44.4 & 38.9 & 16.7 & 2.28 & 0.735 & \\
\hline & & Students & $\mathrm{F}$ & 68 & 78 & 63 & 200 & & \\
\hline & & & $\%$ & 32.5 & 37.3 & 30.1 & 2.02 & 0.793 & 13 \\
\hline & & Total & $\mathrm{F}$ & 108 & 113 & 78 & 210 & 078 & \\
\hline & & & $\%$ & 36.1 & 37.8 & 26.1 & 2.10 & 0.784 & \\
\hline
\end{tabular}




\begin{tabular}{|c|c|c|c|c|c|c|c|c|c|}
\hline \multirow[t]{2}{*}{ No. } & \multirow{2}{*}{\multicolumn{2}{|c|}{ Item }} & \multicolumn{4}{|c|}{ Agreement } & \multirow{2}{*}{$\begin{array}{l}\text { Arithmetic } \\
\text { mean }\end{array}$} & \multirow{2}{*}{$\begin{array}{l}\text { Standard } \\
\text { deviation }\end{array}$} & \multirow{2}{*}{ Rank } \\
\hline & & & & Agree & Undecided & Disagree & & & \\
\hline \multirow[t]{6}{*}{2} & \multirow{6}{*}{$\begin{array}{l}\text { Prohibition of good things and } \\
\text { abandonment of necessities }\end{array}$} & \multirow[t]{2}{*}{ Workers } & $\mathrm{F}$ & 32 & 39 & 19 & \multirow{2}{*}{2.14} & \multirow{2}{*}{0.743} & \multirow{6}{*}{14} \\
\hline & & & $\%$ & 35.6 & 43.3 & 21.1 & & & \\
\hline & & \multirow[t]{2}{*}{ Students } & $\mathrm{F}$ & 56 & 92 & 61 & \multirow{2}{*}{1.98} & \multirow{2}{*}{0.750} & \\
\hline & & & $\%$ & 26.8 & 44.0 & 29.2 & & & \\
\hline & & \multirow[t]{2}{*}{ Total } & $\mathrm{F}$ & 88 & 131 & 80 & \multirow[b]{2}{*}{2.03} & \multirow{2}{*}{0.750} & \\
\hline & & & $\%$ & 29.4 & 43.8 & 26.8 & & & \\
\hline \multirow[t]{6}{*}{18} & \multirow{6}{*}{$\begin{array}{l}\text { Exaggeration and extremism in } \\
\text { all life aspects }\end{array}$} & \multirow[t]{2}{*}{ Workers } & $\mathrm{F}$ & 40 & 30 & 20 & \multirow{2}{*}{2.22} & \multirow{2}{*}{0.790} & \multirow{6}{*}{15} \\
\hline & & & $\%$ & 44.4 & 33.3 & 22.2 & & & \\
\hline & & \multirow[t]{2}{*}{ Students } & $\mathrm{F}$ & 59 & 73 & 77 & & & \\
\hline & & & $\%$ & 28.2 & 34.9 & 36.8 & 1.91 & 0.804 & \\
\hline & & Total & $\mathrm{F}$ & 99 & 103 & 97 & & & \\
\hline & & & $\%$ & 33.1 & 34.4 & 32.4 & 2.01 & 0.811 & \\
\hline 4 & Justifying bloodshed and & Workers & $\mathrm{F}$ & 41 & 29 & 20 & & & \\
\hline & charging people of unbelief & & $\%$ & 45.6 & 32.2 & 22.2 & 2.23 & 0.794 & \\
\hline & & Students & $\mathrm{F}$ & 60 & 55 & 94 & 84 & 8445 & 16 \\
\hline & & & $\%$ & 28.7 & 26.3 & 45.0 & 1.84 & 0.845 & 10 \\
\hline & & Total & $\mathrm{F}$ & 101 & 84 & 114 & 106 & 0.848 & \\
\hline & & & $\%$ & 33.8 & 28.1 & 38.1 & 1.90 & 0.040 & \\
\hline 15 & Underestimating the importance & Workers & $\mathrm{F}$ & 38 & 36 & 16 & & & \\
\hline & of national events & & $\%$ & 42.2 & 40.0 & 17.8 & 2.24 & 0.739 & \\
\hline & & Students & $\mathrm{F}$ & 50 & 71 & 88 & & & \\
\hline & & & $\%$ & 23.9 & 34.0 & 42.1 & 1.82 & 0.794 & 17 \\
\hline & & Total & $\mathrm{F}$ & 88 & 107 & 104 & & 80 & \\
\hline & & & $\%$ & 29.4 & 35.8 & 34.8 & 1.95 & 0.801 & \\
\hline 16 & Refusing to engage in national & Workers & $\mathrm{F}$ & 32 & 38 & 20 & & & \\
\hline & events & & $\%$ & 35.6 & 42.2 & 22.2 & 2.13 & 0.753 & \\
\hline & & Students & $\mathrm{F}$ & 49 & 70 & 90 & & & 18 \\
\hline & & & $\%$ & 23.4 & 33.5 & 43.1 & 1.80 & 0.793 & 18 \\
\hline & & Total & $\mathrm{F}$ & 81 & 108 & 110 & & & \\
\hline & & & $\%$ & 27.1 & 36.1 & 36.8 & 1.90 & 0.795 & \\
\hline 17 & Calling for and promoting anti- & Workers & $\mathrm{F}$ & 34 & 25 & 31 & 2.03 & 0.854 & \\
\hline & national groups & & $\%$ & 37.8 & 27.8 & 34.4 & 2.03 & 0.054 & \\
\hline & & Students & $\mathrm{F}$ & 58 & 49 & 102 & & 0.851 & \\
\hline & & & $\%$ & 27.8 & 23.4 & 48.8 & 1.79 & 0.851 & 19 \\
\hline & & Total & F & 92 & 74 & 133 & & & \\
\hline & & & $\%$ & 30.8 & 24.7 & 44.5 & 1.86 & 0.858 & \\
\hline Ove & rall mean & & & & & & 2.18 & 0.7 & \\
\hline
\end{tabular}

Table (3) shows that the arithmetic means of intellectual delinquencies among youth ranged (2.56:1.86). While (fanaticism) was ranked first, (calling for and promoting anti-national groups) was ranked last.

1. To answer the second question, the frequencies, percentages, arithmetic means, standard deviations, and ranks of the responses to the factors of intellectual delinquency among youth were calculated.

Table 4: Responses of the participants to the family factors of intellectual delinquency among youth

\begin{tabular}{|c|c|c|c|c|c|c|c|c|c|}
\hline \multirow[t]{2}{*}{ No. } & \multirow{2}{*}{\multicolumn{2}{|c|}{ Item }} & \multicolumn{4}{|c|}{ Agreement } & \multirow{2}{*}{$\begin{array}{c}\text { Arithmetic } \\
\text { mean }\end{array}$} & \multirow{2}{*}{$\begin{array}{l}\text { Standard } \\
\text { deviation }\end{array}$} & \multirow{2}{*}{ Rank } \\
\hline & & & & Agree & Undecided & Disagree & & & \\
\hline \multirow[t]{6}{*}{4} & \multirow[t]{6}{*}{ Lack of family dialog } & \multirow[t]{2}{*}{ Workers } & $\mathrm{F}$ & 67 & 22 & 1 & \multirow{2}{*}{2.73} & \multirow{2}{*}{0.469} & \multirow{6}{*}{1} \\
\hline & & & $\%$ & 74.4 & 24.4 & 1.1 & & & \\
\hline & & \multirow[t]{2}{*}{ Students } & $\mathrm{F}$ & 142 & 57 & 10 & \multirow{2}{*}{2.63} & \multirow{2}{*}{0.574} & \\
\hline & & & $\%$ & 67.9 & 27.3 & 4.8 & & & \\
\hline & & \multirow[t]{2}{*}{ Total } & $\mathrm{F}$ & 209 & 79 & 11 & \multirow{2}{*}{2.66} & \multirow{2}{*}{0.546} & \\
\hline & & & $\%$ & 69.9 & 26.4 & 3.7 & & & \\
\hline \multirow[t]{6}{*}{1} & \multirow[t]{6}{*}{ Weak family monitoring of children } & \multirow[t]{2}{*}{ Workers } & $\mathrm{F}$ & 66 & 22 & 2 & \multirow{2}{*}{2.71} & \multirow{2}{*}{0.503} & \multirow{6}{*}{2} \\
\hline & & & $\%$ & 73.3 & 24.4 & 2.2 & & & \\
\hline & & \multirow[t]{2}{*}{ Students } & $\mathrm{F}$ & 137 & 56 & 16 & \multirow{2}{*}{2.58} & \multirow{2}{*}{0.631} & \\
\hline & & & $\%$ & 65.6 & 26.8 & $7 \cdot 7$ & & & \\
\hline & & \multirow[t]{2}{*}{ Total } & $\mathrm{F}$ & 203 & 78 & 18 & \multirow{2}{*}{2.62} & \multirow{2}{*}{0.598} & \\
\hline & & & $\%$ & 67.9 & 26.1 & 6.0 & & & \\
\hline \multirow[t]{4}{*}{9} & \multirow{4}{*}{$\begin{array}{l}\text { Lack of parental understanding of children's } \\
\text { problems and solving them }\end{array}$} & \multirow[t]{2}{*}{ Workers } & $\mathrm{F}$ & 60 & 27 & 3 & \multirow{2}{*}{2.63} & \multirow{2}{*}{0.550} & \\
\hline & & & $\%$ & 66.7 & 30.0 & $3 \cdot 3$ & & & \\
\hline & & Students & $F$ & 134 & 60 & 15 & & 0602 & 3 \\
\hline & & & $\%$ & 64.1 & 28.7 & 7.2 & 2.57 & 0.625 & \\
\hline
\end{tabular}




\begin{tabular}{|c|c|c|c|c|c|c|c|c|c|}
\hline \multirow[t]{4}{*}{ No. 1} & \multirow[t]{4}{*}{ Item } & & \multicolumn{4}{|c|}{ Agreement } & \multirow{2}{*}{$\begin{array}{c}\text { Arithmetic } \\
\text { mean }\end{array}$} & \multirow{2}{*}{$\begin{array}{l}\text { Standard } \\
\text { deviation }\end{array}$} & \multirow{4}{*}{ Rank } \\
\hline & & & & Agree & Undecided & Disagree & & & \\
\hline & & \multirow[t]{2}{*}{ Total } & $\mathrm{F}$ & 194 & 87 & 18 & \multirow{2}{*}{2.59} & \multirow{2}{*}{0.603} & \\
\hline & & & $\%$ & 64.9 & 29.1 & 0.6 & & & \\
\hline \multirow[t]{6}{*}{10} & \multirow{6}{*}{$\begin{array}{l}\text { Parents' preoccupation, making children feel of } \\
\text { isolation }\end{array}$} & \multirow{2}{*}{ Workers } & $\mathrm{F}$ & 72 & 16 & 2 & \multirow{2}{*}{2.78} & \multirow{2}{*}{0.469} & \\
\hline & & & $\%$ & 80.0 & 17.8 & 2.2 & & & \\
\hline & & \multirow[t]{2}{*}{\begin{tabular}{|l|} 
Students \\
\end{tabular}} & $\mathrm{F}$ & 125 & 65 & 19 & 251 & 0650 & \\
\hline & & & $\%$ & 59.8 & 31.1 & 9.1 & $2.3^{1}$ & 0.059 & 4 \\
\hline & & Total & $\mathrm{F}$ & 197 & 81 & 21 & & 0.620 & \\
\hline & & & $\%$ & 65.9 & 27.1 & 7.0 & 2.59 & 0.020 & \\
\hline 5 & Frequent family conflicts & Workers & $\mathrm{F}$ & 60 & 29 & 1 & 266 & & \\
\hline & & & $\%$ & 66.7 & 32.2 & 1.1 & 2.00 & 0.501 & \\
\hline & & Students & $\mathrm{F}$ & 124 & 71 & 14 & & & \\
\hline & & & $\%$ & $59 \cdot 3$ & 34.0 & 6.7 & 2.33 & 0.021 & 3 \\
\hline & & Total & $\mathrm{F}$ & 184 & 100 & 15 & & 0.580 & \\
\hline & & & $\%$ & 61.5 & 33.4 & 5.0 & 2.57 & 0.509 & \\
\hline 7 & The family's lack of interest in children's & Workers & $\mathrm{F}$ & 57 & 28 & 5 & & & \\
\hline & commitment to religious duties & & $\%$ & 63.3 & 31.1 & 5.6 & 2.58 & 0.599 & \\
\hline & & Students & $\mathrm{F}$ & 129 & 62 & 18 & & 0.651 & 6 \\
\hline & & & $\%$ & 61.7 & 29.7 & 8.6 & 2.53 & 0.051 & 0 \\
\hline & & Total & $\mathrm{F}$ & 186 & 90 & 23 & 2.55 & 0.625 & \\
\hline & & & $\%$ & 62.2 & 30.1 & 7.7 & 2.35 & 0.035 & \\
\hline 2 & Lack of good role models in the family & Workers & $\mathrm{F}$ & 58 & 25 & 7 & 2.57 & 0.637 & \\
\hline & & & $\%$ & 64.4 & 27.8 & 7.8 & 2.57 & 0.037 & \\
\hline & & Students & $\mathrm{F}$ & 127 & 64 & 18 & & $0_{5}$ & \\
\hline & & & $\%$ & 60.8 & 30.6 & 8.6 & 2.52 & 0.051 & 7 \\
\hline & & Total & $\mathrm{F}$ & 185 & 89 & 25 & & 66 & \\
\hline & & & $\%$ & 61.9 & 29.8 & 8.4 & 2.54 & 0.040 & \\
\hline 12 & Poor family role in communication with family & Workers & $\mathrm{F}$ & 69 & 18 & 3 & 2.73 & 0.515 & \\
\hline & members & & $\%$ & 76.7 & 20.0 & 3.3 & 2.73 & 0.515 & \\
\hline & & Students & $\mathrm{F}$ & 109 & 79 & 21 & 2,42 & 668 & 8 \\
\hline & & & $\%$ & 52.2 & 37.8 & 10.0 & $2.4^{2}$ & 0.008 & 8 \\
\hline & & Total & $\mathrm{F}$ & 178 & 97 & 24 & 2.52 & 0.642 & \\
\hline & & & $\%$ & 59.5 & 32.4 & 8.0 & $2.5^{2}$ & $0.042^{2}$ & \\
\hline 8 & Aggression with children & Workers & $\mathrm{F}$ & 56 & 28 & 6 & 256 & 0.620 & \\
\hline & & & $\%$ & 62.2 & 31.1 & 6.7 & 2.50 & 0.020 & \\
\hline & & Students & $\mathrm{F}$ & 122 & 61 & 26 & 2,46 & 0.707 & 9 \\
\hline & & & $\%$ & 58.4 & 29.2 & 12.4 & 2.40 & 0.707 & 9 \\
\hline & & Total & $\mathrm{F}$ & 178 & 89 & 32 & 240 & 0.682 & \\
\hline & & & $\%$ & $59 \cdot 5$ & 29.8 & 10.7 & 2.49 & 0.002 & \\
\hline 11 & Poor knowledge of the contemporary updates & Workers & $\mathrm{F}$ & 59 & 26 & 5 & 26 & -006 & \\
\hline & among parents & & $\%$ & 65.6 & 28.9 & 5.6 & 2.60 & 0.590 & \\
\hline & & Students & $\mathrm{F}$ & 107 & 79 & 23 & 240 & 068 & 10 \\
\hline & & & $\%$ & 51.2 & 37.8 & 11.0 & 2.40 & 0.000 & 10 \\
\hline & & Total & $\mathrm{F}$ & 166 & 105 & 28 & 246 & 066 & \\
\hline & & & $\%$ & 55.5 & 35.1 & 9.4 & 2.40 & 0.001 & \\
\hline 6 & Univolved parenting & Workers & $\mathrm{F}$ & 50 & 35 & 5 & 250 & 0604 & \\
\hline & & & $\%$ & 55.6 & 38.9 & 5.6 & 2.50 & 0.004 & \\
\hline & & Students & $\mathrm{F}$ & 105 & 77 & 27 & 2.37 & 0.703 & 11 \\
\hline & & & $\%$ & 50.2 & 36.8 & 12.9 & 2.31 & & \\
\hline & & Total & $\mathrm{F}$ & 155 & 112 & 32 & 2.41 & 0.677 & \\
\hline & & & $\%$ & 51.8 & 37.5 & 10.7 & 2.41 & 0.077 & \\
\hline 3 & Aggressive treatment of youth & Workers & $\mathrm{F}$ & 49 & 28 & 13 & 2,40 & 0.731 & \\
\hline & & & $\%$ & 54.4 & 31.1 & 14.4 & 2.40 & 0.731 & \\
\hline & & Students & $\mathrm{F}$ & 110 & 73 & 26 & 240 & 070 & \\
\hline & & & $\%$ & 52.6 & 34.9 & 12.4 & 2.40 & 0.701 & 12 \\
\hline & & Total & $\mathrm{F}$ & 159 & 101 & 39 & 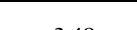 & - 78 & \\
\hline & & & $\%$ & 53.2 & 33.8 & 13.0 & 2.40 & 0.709 & \\
\hline Ove & rall mean & & & & & & 2.53 & 0.533 & \\
\hline
\end{tabular}

Table (4) illustrates that the arithmetic means of family factors of intellectual delinquency among youth ranged (2.66:2.40). While (lack of family dialog) was ranked first, (aggressive treatment of youth) was ranked last. Among social workers, (parents' preoccupation) was ranked first. 
Table 5: Responses of the participants to the societal factors of intellectual delinquency among youth

\begin{tabular}{|c|c|c|c|c|c|c|c|c|c|}
\hline \multirow[t]{2}{*}{ No. } & \multirow{2}{*}{\multicolumn{2}{|c|}{ Item }} & \multicolumn{4}{|c|}{ Agreement } & \multirow{2}{*}{$\begin{array}{c}\text { Arithmetic } \\
\text { mean }\end{array}$} & \multirow{2}{*}{$\begin{array}{l}\text { Standard } \\
\text { deviation }\end{array}$} & \multirow{2}{*}{ Rank } \\
\hline & & & & Agree & Undecided & Disagree & & & \\
\hline \multirow[t]{6}{*}{4} & \multirow{6}{*}{$\begin{array}{l}\text { Youth's lack of knowledge } \\
\text { about the legal means } \\
\text { that deter delinquents }\end{array}$} & \multirow[t]{2}{*}{ Workers } & $\mathrm{F}$ & 63 & 21 & 6 & \multirow{2}{*}{2.63} & \multirow{2}{*}{0.608} & \multirow{6}{*}{1} \\
\hline & & & $\%$ & 70.0 & $23 \cdot 3$ & 6.7 & & & \\
\hline & & \multirow[t]{2}{*}{ Students } & $\mathrm{F}$ & 148 & 47 & 14 & \multirow{2}{*}{2.64} & & \\
\hline & & & $\%$ & 70.8 & 22.5 & 6.7 & & 0.605 & \\
\hline & & Total & $\mathrm{F}$ & 211 & 68 & 20 & 260 & $60-$ & \\
\hline & & & $\%$ & 70.6 & 22.7 & 6.7 & 2.64 & 0.605 & \\
\hline 2 & Unemployment & Workers & $\mathrm{F}$ & 59 & 29 & 2 & & & \\
\hline & & & $\%$ & 65.6 & 32.2 & 2.2 & 2.63 & 0.529 & \\
\hline & & Students & F & 140 & 46 & 23 & & & \\
\hline & & & $\%$ & 67.0 & 22.0 & 11.0 & 2.56 & 0.685 & 2 \\
\hline & & Total & $\mathrm{F}$ & 199 & 75 & 25 & 258 & 0.642 & \\
\hline & & & $\%$ & 66.6 & 25.1 & 8.4 & 2.58 & 0.642 & \\
\hline 1 & Lack of successful societal & Workers & $\mathrm{F}$ & 65 & 23 & 2 & & & \\
\hline & systems to handle youth & & $\%$ & 72.2 & 25.6 & 2.2 & 2.70 & 0.507 & \\
\hline & & Students & $\mathrm{F}$ & 124 & 68 & 17 & & & \\
\hline & & & $\%$ & $59 \cdot 3$ & 32.5 & 8.1 & 2.51 & 0.644 & 3 \\
\hline & & Total & $\mathrm{F}$ & 189 & 91 & 19 & & 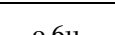 & \\
\hline & & & $\%$ & 63.2 & 30.4 & 6.4 & 2.57 & 0.611 & \\
\hline 3 & Dissemination and ease of & Workers & $\mathrm{F}$ & 58 & 26 & 6 & & 618 & \\
\hline & access to the & & $\%$ & 64.4 & 28.9 & 6.7 & 2.58 & 0.618 & \\
\hline & pornographic culture via & Students & $\mathrm{F}$ & 140 & 48 & 21 & & 0 670 & \\
\hline & & & $\%$ & 67.0 & 23.0 & 10.0 & 2.57 & 0.670 & 4 \\
\hline & & Total & $\mathrm{F}$ & 198 & 74 & 27 & & & \\
\hline & & & $\%$ & 66.2 & 24.7 & 9.0 & 2.57 & 0.653 & \\
\hline 6 & Invalid preventive role of & Workers & $\mathrm{F}$ & 73 & 15 & 2 & & 0462 & \\
\hline & social institutions & & $\%$ & 81.1 & 16.7 & 2.2 & 2.79 & 0.402 & \\
\hline & & Students & $\mathrm{F}$ & 120 & 66 & 23 & & 686 & \\
\hline & & & $\%$ & 57.4 & 31.6 & 11.0 & 2.46 & 0.686 & 5 \\
\hline & & Total & $\mathrm{F}$ & 193 & 81 & 25 & 256 & 064 & \\
\hline & & & $\%$ & 64.5 & 27.1 & 8.4 & 2.56 & 0.644 & \\
\hline 5 & Role of media and social & Workers & $\mathrm{F}$ & 67 & 22 & 1 & & 0460 & \\
\hline & media in intellectual & & $\%$ & 74.4 & 24.4 & 1.1 & 2.73 & 0.469 & \\
\hline & delinquencies & Students & $\mathrm{F}$ & 125 & 60 & 24 & & & 6 \\
\hline & & & $\%$ & 59.8 & 28.7 & 11.5 & 2.48 & 0.694 & 6 \\
\hline & & Total & $\mathrm{F}$ & 192 & 82 & 25 & 256 & 64 & \\
\hline & & & $\%$ & 64.2 & 27.4 & 8.4 & 2.56 & 0.644 & \\
\hline 8 & Lack of entertainment & Workers & $\mathrm{F}$ & 58 & 24 & 8 & & & \\
\hline & means & & $\%$ & 64.4 & 26.7 & 8.9 & 2.56 & 0.655 & \\
\hline & & Students & $\mathrm{F}$ & 121 & 67 & 21 & & & \\
\hline & & & $\%$ & 57.9 & 32.1 & 10.0 & 2.48 & 0.673 & 7 \\
\hline & & Total & $\mathrm{F}$ & 179 & 91 & 29 & 50 & 0667 & \\
\hline & & & $\%$ & 59.9 & 30.4 & $9 \cdot 7$ & 2.50 & 0.007 & \\
\hline 12 & Poor control of media and & Workers & $\mathrm{F}$ & 62 & 23 & 5 & 26 & -8 & \\
\hline & social media & & $\%$ & 68.9 & 25.6 & 5.6 & 2.63 & 0.589 & \\
\hline & & Students & $\mathrm{F}$ & 117 & 66 & 26 & 4 & 70- & 8 \\
\hline & & & $\%$ & 56.0 & 31.6 & 12.4 & 2.44 & 0.705 & 8 \\
\hline & & Total & $\mathrm{F}$ & 179 & 89 & 31 & & 0667 & \\
\hline & & & $\%$ & $59 \cdot 9$ & 29.8 & 10.4 & 2.49 & 0.667 & \\
\hline 9 & Overlooking the needs of & Workers & $\mathrm{F}$ & 54 & 31 & 5 & & 0.602 & \\
\hline & youth in the national & & $\%$ & 60.0 & 34.4 & 5.6 & 2.54 & 0.603 & \\
\hline & strategic plans & Students & $\mathrm{F}$ & 107 & 78 & 24 & 40 & 087 & 0 \\
\hline & & & $\%$ & 51.2 & $37 \cdot 3$ & 11.5 & 2.40 & & 9 \\
\hline & & Total & $\mathrm{F}$ & 161 & 109 & 29 & & ०66 & \\
\hline & & & $\%$ & 53.8 & 36.5 & 9.7 & 2.44 & 0.665 & \\
\hline 7 & Attracting youth to gain & Workers & $\mathrm{F}$ & 61 & 23 & 6 & 26 & ביא & \\
\hline & money quickly by joining & & $\%$ & 67.8 & 25.6 & 6.7 & 2.01 & 0.012 & 10 \\
\hline & extreme groups & Students & $\mathrm{F}$ & 109 & 65 & 35 & & & 10 \\
\hline & & & $\%$ & 52.2 & 31.1 & 16.7 & 2.35 & 0.753 & \\
\hline
\end{tabular}




\begin{tabular}{|c|c|c|c|c|c|c|c|c|c|}
\hline \multirow[t]{4}{*}{ No. } & \multirow[t]{4}{*}{ Item } & & \multicolumn{4}{|c|}{ Agreement } & \multirow{2}{*}{$\begin{array}{c}\text { Arithmetic } \\
\text { mean }\end{array}$} & \multirow{2}{*}{$\begin{array}{l}\text { Standard } \\
\text { deviation } \\
\end{array}$} & \multirow{4}{*}{ Rank } \\
\hline & & & & Agree & Undecided & Disagree & & & \\
\hline & & \multirow[t]{2}{*}{ Total } & $\mathrm{F}$ & 170 & 88 & 41 & \multirow[b]{2}{*}{2.43} & \multirow[b]{2}{*}{0.722} & \\
\hline & & & $\%$ & 56.9 & 29.4 & 13.7 & & & \\
\hline \multirow[t]{6}{*}{10} & \multirow{6}{*}{$\begin{array}{l}\text { Strong impact of extreme } \\
\text { groups on youth }\end{array}$} & \multirow[t]{2}{*}{ Workers } & $\mathrm{F}$ & 55 & 29 & 6 & \multirow[b]{2}{*}{2.54} & \multirow{2}{*}{0.621} & \multirow{6}{*}{11} \\
\hline & & & $\%$ & 61.1 & 32.2 & 6.7 & & & \\
\hline & & \multirow[t]{2}{*}{ Students } & $\mathrm{F}$ & 95 & 75 & 39 & \multirow{2}{*}{2.27} & \multirow{2}{*}{0.756} & \\
\hline & & & $\%$ & 45.5 & 35.9 & 18.7 & & & \\
\hline & & \multirow[t]{2}{*}{ Total } & $\mathrm{F}$ & 150 & 104 & 45 & \multirow{2}{*}{2.35} & \multirow{2}{*}{0.728} & \\
\hline & & & $\%$ & 50.2 & 34.8 & 15.1 & & & \\
\hline \multirow[t]{6}{*}{11} & \multirow{6}{*}{$\begin{array}{l}\text { Weak citizenship among } \\
\text { youth }\end{array}$} & \multirow[t]{2}{*}{ Workers } & $\mathrm{F}$ & 52 & 29 & 9 & \multirow{2}{*}{2.48} & \multirow{2}{*}{0.674} & \multirow{6}{*}{12} \\
\hline & & & $\%$ & 57.8 & 32.2 & 10.0 & & & \\
\hline & & \multirow[t]{2}{*}{ Students } & $\mathrm{F}$ & 73 & 96 & 40 & \multirow{2}{*}{2.16} & \multirow{2}{*}{0.720} & \\
\hline & & & $\%$ & 34.9 & 45.9 & 19.1 & & & \\
\hline & & \multirow[t]{2}{*}{ Total } & $\mathrm{F}$ & 125 & 125 & 49 & \multirow{2}{*}{2.25} & \multirow{2}{*}{0.720} & \\
\hline & & & $\%$ & 41.8 & 41.8 & 16.4 & & & \\
\hline \multicolumn{7}{|c|}{ Overall mean } & 2.50 & \\
\hline
\end{tabular}

Table (5) shows that the arithmetic means of societal factors of intellectual delinquency among youth ranged (2.64:2.25). While (youth's lack of knowledge about the legal means that deter delinquents) was ranked first, (weak citizenship among youth) was ranked last. Social workers agreed that (invalid preventive role of social institutions) was ranked first. The participant students agreed that (youth's lack of knowledge about the legal means that deter delinquents) was ranked first.

Table 6: Responses of the participants to the personal factors of intellectual delinquency among youth

\begin{tabular}{|c|c|c|c|c|c|c|c|c|c|}
\hline \multirow[t]{2}{*}{ No. } & \multirow{2}{*}{\multicolumn{2}{|c|}{ Item }} & \multicolumn{4}{|c|}{ Agreement } & \multirow{2}{*}{$\begin{array}{l}\text { Arithmetic } \\
\text { mean }\end{array}$} & \multirow{2}{*}{$\begin{array}{l}\text { Standard } \\
\text { deviation }\end{array}$} & \multirow{2}{*}{ Rank } \\
\hline & & & & Agree & Undecided & Disagree & & & \\
\hline \multirow[t]{6}{*}{9} & \multirow{6}{*}{$\begin{array}{l}\text { Weak personality, docileness, } \\
\text { and being influenced by } \\
\text { others }\end{array}$} & \multirow[t]{2}{*}{ Workers } & $\mathrm{F}$ & 65 & 24 & 1 & \multirow{2}{*}{2.71} & \multirow{2}{*}{0.480} & \multirow{6}{*}{1} \\
\hline & & & $\%$ & 72.2 & 26.7 & 1.1 & & & \\
\hline & & \multirow[t]{2}{*}{ Students } & $\mathrm{F}$ & 142 & 56 & 11 & \multirow{2}{*}{2.63} & \multirow{2}{*}{0.584} & \\
\hline & & & $\%$ & 67.9 & 26.8 & $5 \cdot 3$ & & & \\
\hline & & \multirow[t]{2}{*}{ Total } & $\mathrm{F}$ & 207 & 80 & 12 & \multirow{2}{*}{2.65} & \multirow{2}{*}{0.555} & \\
\hline & & & $\%$ & 69.2 & 26.8 & 4.0 & & & \\
\hline \multirow[t]{6}{*}{6} & \multirow[t]{6}{*}{ Blind imitation of others } & \multirow[t]{2}{*}{ Workers } & $\mathrm{F}$ & 58 & 30 & 2 & \multirow{2}{*}{2.62} & \multirow{2}{*}{0.532} & \multirow{6}{*}{2} \\
\hline & & & $\%$ & 64.4 & 33.3 & 2.2 & & & \\
\hline & & \multirow[t]{2}{*}{ Students } & $\mathrm{F}$ & 144 & 53 & 12 & \multirow{2}{*}{2.63} & \multirow{2}{*}{0.591} & \\
\hline & & & $\%$ & 68.9 & 25.4 & 5.7 & & & \\
\hline & & \multirow[t]{2}{*}{ Total } & $\mathrm{F}$ & 202 & 83 & 14 & 263 & & \\
\hline & & & $\%$ & 67.6 & 27.8 & 4.7 & 2.03 & 0.573 & \\
\hline 11 & Lack of personal awareness & Workers & $\mathrm{F}$ & 62 & 24 & 4 & 264 & 0.567 & \\
\hline & & & $\%$ & 68.9 & 26.7 & 4.4 & 2.04 & 0.507 & \\
\hline & & Students & $\mathrm{F}$ & 140 & 55 & 14 & 260 & 0.612 & \\
\hline & & & $\%$ & 67.0 & 26.3 & 6.7 & 2.00 & 0.013 & 3 \\
\hline & & Total & $\mathrm{F}$ & 202 & 79 & 18 & 262 & & \\
\hline & & & $\%$ & 67.6 & 26.4 & 6.0 & 2.02 & 0.599 & \\
\hline 8 & Gaining knowledge from & Workers & $\mathrm{F}$ & 67 & 19 & 4 & & & \\
\hline & suspicious sources & & $\%$ & 74.4 & 21.1 & 4.4 & 2.70 & 0.550 & \\
\hline & & Students & $\mathrm{F}$ & 136 & 57 & 16 & & 0.632 & \\
\hline & & & $\%$ & 65.1 & 27.3 & $7 \cdot 7$ & 2.57 & 0.032 & 4 \\
\hline & & Total & $\mathrm{F}$ & 203 & 76 & 20 & 263 & & \\
\hline & & & $\%$ & 67.9 & 25.4 & 6.7 & 2.01 & 0.010 & \\
\hline 7 & Lack of experience and & Workers & $\mathrm{F}$ & 66 & 19 & 5 & 2.68 & & \\
\hline & wisdom & & $\%$ & 73.3 & 21.1 & 5.6 & 2.08 & 0.577 & \\
\hline & & Students & $\mathrm{F}$ & 135 & 58 & 16 & 2.57 & 0.633 & 5 \\
\hline & & & $\%$ & 64.6 & 27.8 & $7 \cdot 7$ & 2.57 & 0.033 & 3 \\
\hline & & Total & $\mathrm{F}$ & 201 & 77 & 21 & 2.60 & 0.618 & \\
\hline & & & $\%$ & 67.2 & 25.8 & 7.0 & & & \\
\hline
\end{tabular}




\begin{tabular}{|c|c|c|c|c|c|c|c|c|c|}
\hline \multirow[t]{2}{*}{ No. } & \multirow{2}{*}{\multicolumn{2}{|c|}{ Item }} & \multicolumn{4}{|c|}{ Agreement } & \multirow{2}{*}{$\begin{array}{c}\text { Arithmetic } \\
\text { mean }\end{array}$} & \multirow{2}{*}{$\begin{array}{l}\text { Standard } \\
\text { deviation }\end{array}$} & \multirow{2}{*}{ Rank } \\
\hline & & & & Agree & Undecided & Disagree & & & \\
\hline \multirow[t]{6}{*}{1} & \multirow[t]{6}{*}{ Despair and frustration } & \multirow[t]{2}{*}{ Workers } & $\mathrm{F}$ & 63 & 25 & 2 & \multirow{2}{*}{2.68} & \multirow{2}{*}{0.516} & \multirow{6}{*}{6} \\
\hline & & & $\%$ & 70.0 & 27.8 & 2.2 & & & \\
\hline & & \multirow[t]{2}{*}{ Students } & $\mathrm{F}$ & 126 & 67 & 16 & \multirow{2}{*}{2.53} & \multirow{2}{*}{0.636} & \\
\hline & & & $\%$ & 60.3 & 32.1 & $7 \cdot 7$ & & & \\
\hline & & \multirow[t]{2}{*}{ Total } & $\mathrm{F}$ & 189 & 92 & 18 & \multirow{2}{*}{2.57} & \multirow{2}{*}{0.605} & \\
\hline & & & $\%$ & 63.2 & 30.8 & 6.0 & & & \\
\hline \multirow[t]{6}{*}{4} & \multirow{6}{*}{$\begin{array}{l}\text { Psychological and social } \\
\text { isolation from family and } \\
\text { society }\end{array}$} & \multirow[t]{2}{*}{ Workers } & $\mathrm{F}$ & 57 & 28 & 5 & \multirow{2}{*}{2.58} & & \\
\hline & & & $\%$ & 63.3 & 31.1 & 5.6 & & 0.599 & \\
\hline & & Students & $\mathrm{F}$ & 116 & 76 & 17 & & & \\
\hline & & & $\%$ & $55 \cdot 5$ & 36.4 & 8.1 & 2.47 & 0.643 & 7 \\
\hline & & Total & $\mathrm{F}$ & 173 & 104 & 22 & & & \\
\hline & & & $\%$ & 57.9 & 34.8 & $7 \cdot 4$ & 2.51 & 0.031 & \\
\hline 10 & Lack of life skills & Workers & $\mathrm{F}$ & 53 & 31 & 6 & & & \\
\hline & & & $\%$ & 58.9 & 34.4 & 6.7 & $2.5^{2}$ & 0.022 & \\
\hline & & Students & $\mathrm{F}$ & 118 & 76 & 15 & & 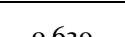 & 8 \\
\hline & & & $\%$ & 56.5 & 36.4 & 7.2 & 2.49 & 0.629 & 8 \\
\hline & & Total & $\mathrm{F}$ & 171 & 107 & 21 & & 0606 & \\
\hline & & & $\%$ & 57.2 & 35.8 & 7.0 & 2.50 & 0.626 & \\
\hline 5 & Low self-confidence & Workers & $\mathrm{F}$ & 54 & 31 & 5 & & & \\
\hline & & & $\%$ & 60.0 & 34.4 & 5.6 & 2.54 & 0.603 & \\
\hline & & Students & $\mathrm{F}$ & 117 & 75 & 17 & & & \\
\hline & & & $\%$ & 56.0 & 35.9 & 8.1 & 2.48 & 0.644 & 9 \\
\hline & & Total & $\mathrm{F}$ & 171 & 106 & 22 & & & \\
\hline & & & $\%$ & 57.2 & $35 \cdot 5$ & 7.4 & 2.50 & 0.031 & \\
\hline 2 & Fanaticism & Workers & $\mathrm{F}$ & 54 & 32 & 4 & & & \\
\hline & & & $\%$ & 60.0 & 35.6 & 4.4 & 2.56 & 0.583 & \\
\hline & & Students & $\mathrm{F}$ & 117 & 71 & 21 & & & \\
\hline & & & $\%$ & 56.0 & 34.0 & 10.0 & 2.46 & 0.672 & 10 \\
\hline & & Total & $\mathrm{F}$ & 171 & 103 & 25 & & & \\
\hline & & & $\%$ & 57.2 & 34.4 & 8.4 & 2.49 & 0.647 & \\
\hline 3 & Adhering to old ideas without & Workers & $\mathrm{F}$ & 52 & 30 & 8 & & 068 & \\
\hline & distinguishing right from & & $\%$ & 57.8 & $33 \cdot 3$ & 8.9 & 2.49 & 0.658 & \\
\hline & wrong ones & Students & $\mathrm{F}$ & 119 & 68 & 22 & 2,16 & 0670 & 1 \\
\hline & & & $\%$ & 56.9 & 32.5 & 10.5 & 2.46 & 0.679 & 11 \\
\hline & & Total & $\mathrm{F}$ & 171 & 98 & 30 & & & \\
\hline & & & $\%$ & 57.2 & 32.8 & 10.0 & 2.47 & 0.672 & \\
\hline 12 & Multiple intellectual & Workers & $\mathrm{F}$ & 45 & 36 & 9 & & ב & \\
\hline & references & & $\%$ & 50.0 & 40.0 & 10.0 & 2.40 & 0.667 & \\
\hline & & Students & $\mathrm{F}$ & 109 & 80 & 20 & & 0667 & 12 \\
\hline & & & $\%$ & 52.2 & 38.3 & 9.6 & 2.43 & 0.662 & 12 \\
\hline & & Total & $\mathrm{F}$ & 154 & 116 & 29 & & 0662 & \\
\hline & & & $\%$ & 51.5 & 38.8 & 9.7 & 2.42 & 0.662 & \\
\hline Ovel & rall mean & & & & & & 2.55 & 0.527 & \\
\hline
\end{tabular}

Table (6) illustrates that the arithmetic means of personal factors of intellectual delinquency among youth ranged (2.65:2.42). While (weak personality, docileness, and being influenced by others) was ranked first, (multiple intellectual references) was ranked last. Social workers agreed that (weak personality, docileness, and being influenced by others) was ranked first. Moreover, the participant students agreed that items (weak personality, docileness, and being influenced by others) and (blind imitation of others) were ranked first. 
Table 7: Responses of the participants to the educational factors of intellectual delinquency among youth

\begin{tabular}{|c|c|c|c|c|c|c|c|c|c|}
\hline \multirow[t]{2}{*}{ No. } & \multirow{2}{*}{\multicolumn{2}{|c|}{ Item }} & \multicolumn{4}{|c|}{ Agreement } & \multirow{2}{*}{$\begin{array}{c}\text { Arithmetic } \\
\text { mean }\end{array}$} & \multirow{2}{*}{$\begin{array}{l}\text { Standard } \\
\text { deviation }\end{array}$} & \multirow{2}{*}{ Rank } \\
\hline & & & & Agree & Undecided & Disagree & & & \\
\hline \multirow[t]{6}{*}{2} & \multirow{6}{*}{$\begin{array}{l}\text { Leniency in applying } \\
\text { disciplinary regulations } \\
\text { and facing student } \\
\text { violations }\end{array}$} & \multirow[t]{2}{*}{ Workers } & F & 52 & 35 & 3 & \multirow{2}{*}{2.54} & \multirow{2}{*}{0.564} & \multirow{6}{*}{1} \\
\hline & & & $\%$ & 57.8 & 38.9 & 3.3 & & & \\
\hline & & \multirow[t]{2}{*}{ Students } & $\mathrm{F}$ & 118 & 65 & 26 & \multirow{2}{*}{2.44} & \multirow{2}{*}{0.705} & \\
\hline & & & $\%$ & 56.5 & 31.1 & 12.4 & & & \\
\hline & & Total & $\mathrm{F}$ & 170 & 100 & 29 & & & \\
\hline & & & $\%$ & 56.9 & 33.4 & 9.7 & 2.47 & 0.667 & \\
\hline 9 & Weak role of the social & Workers & $\mathrm{F}$ & 58 & 28 & 4 & & & \\
\hline & guidance departments in & & $\%$ & 64.4 & 31.1 & 4.4 & 2.60 & 0.577 & \\
\hline & the intellectual & Students & $\mathrm{F}$ & 104 & 83 & 22 & & & \\
\hline & awareness of students & & $\%$ & 49.8 & $39 \cdot 7$ & 10.5 & 2.39 & 0.672 & 2 \\
\hline & & Total & $\mathrm{F}$ & 162 & 111 & 26 & & 60 & \\
\hline & & & $\%$ & 54.2 & 37.1 & 8.7 & 2.45 & 0.651 & \\
\hline 1 & Lack of the faculty's & Workers & $\mathrm{F}$ & 51 & 33 & 6 & & & \\
\hline & interest in raising student & & $\%$ & 56.7 & 36.7 & 6.7 & 2.50 & 0.623 & \\
\hline & awareness about the & Students & $\mathrm{F}$ & 111 & 77 & 21 & & & \\
\hline & & & $\%$ & 53.1 & 36.8 & 10.0 & 2.43 & 0.670 & 3 \\
\hline & & Total & $\mathrm{F}$ & 162 & 110 & 27 & & & \\
\hline & & & $\%$ & 54.2 & 36.8 & 9.0 & 2.45 & 0.656 & \\
\hline 3 & Lack of educational & Workers & $\mathrm{F}$ & 54 & 27 & 9 & & & \\
\hline & activities in the courses & & $\%$ & 60.0 & 30.0 & 10.0 & 2.50 & 0.675 & \\
\hline & that promote moderation & Students & $\mathrm{F}$ & 109 & 78 & 22 & 24 & 675 & \\
\hline & & & $\%$ & 52.2 & $37 \cdot 3$ & 10.5 & 2.42 & 0.075 & 4 \\
\hline & & Total & $\mathrm{F}$ & 163 & 105 & 31 & & & \\
\hline & & & $\%$ & 54.5 & 35.1 & 10.4 & 2.44 & 0.675 & \\
\hline 12 & Different social and & Workers & $\mathrm{F}$ & 39 & 43 & 8 & & 0630 & \\
\hline & economic levels and & & $\%$ & $43 \cdot 3$ & 47.8 & 8.9 & 2.34 & 0.639 & \\
\hline & influence of students on & Students & $\mathrm{F}$ & 111 & 81 & 17 & & & \\
\hline & each other & & $\%$ & 53.1 & 38.8 & 8.1 & 2.45 & 0.642 & 5 \\
\hline & & Total & $\mathrm{F}$ & 150 & 124 & 25 & & & \\
\hline & & & $\%$ & 50.2 & 41.5 & 8.4 & 2.42 & 0.642 & \\
\hline 5 & Weak university role in & Workers & $\mathrm{F}$ & 50 & 33 & 7 & 2,8 & 0.640 & \\
\hline & confronting the moral & & $\%$ & 55.6 & 36.7 & 7.8 & 2.48 & 0.640 & \\
\hline & deviation of students & Students & $\mathrm{F}$ & 100 & 89 & 20 & 2.38 & 0.656 & 6 \\
\hline & & & $\%$ & 47.8 & 42.6 & 9.6 & 2.38 & 0.656 & 6 \\
\hline & & Total & $\mathrm{F}$ & 150 & 122 & 27 & 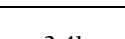 & $6=1$ & \\
\hline & & & $\%$ & 50.2 & 40.8 & 9.0 & 2.41 & 0.651 & \\
\hline 8 & Lack of active university & Workers & $\mathrm{F}$ & 55 & 27 & 8 & & & \\
\hline & policies to promote & & $\%$ & 61.1 & 30.0 & 8.9 & $2.5^{2}$ & 0.657 & \\
\hline & intellectual security & Students & $\mathrm{F}$ & 101 & 83 & 25 & & & \\
\hline & & & $\%$ & 48.3 & 39.7 & 12.0 & 2.36 & 0.688 & 7 \\
\hline & & Total & $\mathrm{F}$ & 156 & 110 & 33 & & 68 & \\
\hline & & & $\%$ & 52.2 & 36.8 & 11.0 & 2.41 & 0.681 & \\
\hline 7 & Lack and weakness of & Workers & $\mathrm{F}$ & 54 & 28 & 8 & & & \\
\hline & extracurricular activities & & $\%$ & 60.0 & 31.1 & 8.9 & 2.51 & 0.658 & \\
\hline & that promote the & Students & $\mathrm{F}$ & 103 & 80 & 26 & & ( 66 & 8 \\
\hline & intellectual security of & & $\%$ & $49 \cdot 3$ & 38.3 & 12.4 & 2.37 & 0.696 & 8 \\
\hline & students & Total & $\mathrm{F}$ & 157 & 108 & 34 & & & \\
\hline & & & $\%$ & 52.5 & 36.1 & 11.4 & 2.41 & 0.686 & \\
\hline 6 & Weak university role in & Workers & $\mathrm{F}$ & 48 & 32 & 10 & & $68-$ & \\
\hline & confronting and solving & & $\%$ & $53 \cdot 3$ & 35.6 & 11.1 & 2.42 & 0.687 & \\
\hline & student problems & Students & $\bar{F}$ & 103 & 81 & 25 & & & \\
\hline & & & $\%$ & $49 \cdot 3$ & 38.8 & 12.0 & 2.37 & 0.690 & 9 \\
\hline & & Total & $\mathrm{F}$ & 151 & 113 & 35 & & 688 & \\
\hline & & & $\%$ & 50.5 & 37.8 & 11.7 & 2.39 & 0.688 & \\
\hline 4 & Weak university role in & Workers & $\mathrm{F}$ & 49 & 35 & 6 & 2,8 & ב26 & 10 \\
\hline & utilizing knowledge and & & $\%$ & 54.4 & 38.9 & 6.7 & 2.48 & 0.622 & 10 \\
\hline
\end{tabular}




\begin{tabular}{|c|c|c|c|c|c|c|c|c|c|}
\hline \multirow[t]{6}{*}{ No. } & \multirow{2}{*}{\multicolumn{2}{|c|}{ Item }} & \multicolumn{4}{|c|}{ Agreement } & \multirow{2}{*}{$\begin{array}{c}\text { Arithmetic } \\
\text { mean }\end{array}$} & \multirow{2}{*}{$\begin{array}{l}\text { Standard } \\
\text { deviation }\end{array}$} & \multirow{6}{*}{ Rank } \\
\hline & & & & Agree & Undecided & Disagree & & & \\
\hline & \multirow{4}{*}{$\begin{array}{l}\text { scientific research to } \\
\text { serve the issues of } \\
\text { national and intellectual } \\
\text { security }\end{array}$} & \multirow[t]{2}{*}{ Students } & $\mathrm{F}$ & 95 & 86 & 28 & \multirow{2}{*}{2.32} & \multirow{2}{*}{0.699} & \\
\hline & & & $\%$ & $45 \cdot 5$ & 41.1 & 13.4 & & & \\
\hline & & \multirow[t]{2}{*}{ Total } & $\mathrm{F}$ & 144 & 121 & 34 & \multirow{2}{*}{2.37} & \multirow{2}{*}{0.679} & \\
\hline & & & $\%$ & 48.2 & 40.5 & 11.4 & & & \\
\hline \multirow[t]{6}{*}{10} & \multirow{6}{*}{$\begin{array}{l}\text { Stress because of the } \\
\text { inability to fit in the } \\
\text { major that the student is } \\
\text { forced to study }\end{array}$} & \multirow[t]{2}{*}{ Workers } & $\mathrm{F}$ & 38 & 40 & 12 & \multirow{2}{*}{2.29} & \multirow{2}{*}{0.691} & \multirow{6}{*}{11} \\
\hline & & & $\%$ & 42.2 & 44.4 & 13.3 & & & \\
\hline & & \multirow[t]{2}{*}{ Students } & $\mathrm{F}$ & 108 & 79 & 22 & \multirow{2}{*}{2.41} & \multirow{2}{*}{0.674} & \\
\hline & & & $\%$ & 51.7 & 37.8 & 10.5 & & & \\
\hline & & \multirow[t]{2}{*}{ Total } & $\mathrm{F}$ & 146 & 119 & 34 & \multirow{2}{*}{2.37} & \multirow{2}{*}{0.681} & \\
\hline & & & $\%$ & 48.8 & 39.8 & 11.4 & & & \\
\hline \multirow[t]{6}{*}{11} & \multirow{6}{*}{$\begin{array}{l}\text { Student cultural } \\
\text { exchange and acquiring } \\
\text { inappropriate ideas }\end{array}$} & \multirow[t]{2}{*}{ Workers } & $\mathrm{F}$ & 41 & 38 & 11 & \multirow{2}{*}{2.33} & \multirow{2}{*}{0.687} & \multirow{6}{*}{12} \\
\hline & & & $\%$ & 45.6 & 42.2 & 12.2 & & & \\
\hline & & \multirow[t]{2}{*}{ Students } & $\mathrm{F}$ & 98 & 90 & 21 & \multirow{2}{*}{2.37} & \multirow{2}{*}{0.660} & \\
\hline & & & $\%$ & 46.9 & 43.1 & 10.0 & & & \\
\hline & & \multirow[t]{2}{*}{ Total } & $\mathrm{F}$ & 139 & 128 & 32 & & 067 & \\
\hline & & & $\%$ & 46.5 & 42.8 & 10.7 & 2.36 & 0.667 & \\
\hline & & Overa & neal & & & & 2.41 & 0.6 & \\
\hline
\end{tabular}

Table (7) shows that the arithmetic means of educational factors of intellectual delinquency among youth ranged (2.74:2.36). While (leniency in applying disciplinary regulations and facing student violations) was ranked first, (student cultural exchange and acquiring inappropriate ideas) was ranked last. Social workers agreed that (weak role of the social guidance departments in the intellectual awareness of students) was ranked first. The participant students agreed that (different social and economic levels and influence of students on each other) was ranked first.

1. To answer the third question, the frequencies, percentages, arithmetic means, standard deviations, and ranks of the responses to the suggested ways of preventing the factors of intellectual delinquencies among youth were calculated.

Table 8: Responses of the participants to the suggested ways of preventing the family factors of intellectual delinquencies among youth

\begin{tabular}{|c|c|c|c|c|c|c|c|c|c|}
\hline \multirow[t]{2}{*}{ No. } & \multirow{2}{*}{\multicolumn{2}{|c|}{ Item }} & \multicolumn{4}{|c|}{ Agreement } & \multirow{2}{*}{$\begin{array}{c}\text { Arithmetic } \\
\text { mean }\end{array}$} & \multirow{2}{*}{$\begin{array}{l}\text { Standard } \\
\text { deviation }\end{array}$} & \multirow{2}{*}{ Rank } \\
\hline & & & & Agree & Undecided & Disagree & & & \\
\hline \multirow[t]{6}{*}{8} & \multirow{6}{*}{$\begin{array}{l}\text { Giving the opportunity } \\
\text { within the family to } \\
\text { exchange opinions and } \\
\text { hear the other's } \\
\text { opinion }\end{array}$} & \multirow[t]{2}{*}{ Workers } & $\mathrm{F}$ & 69 & 21 & - & \multirow{2}{*}{2.77} & \multirow{2}{*}{0.425} & \multirow{6}{*}{1} \\
\hline & & & $\%$ & 76.7 & 23.3 & - & & & \\
\hline & & \multirow[t]{2}{*}{ Students } & $\mathrm{F}$ & 158 & 47 & 4 & \multirow{2}{*}{2.74} & \multirow{2}{*}{0.483} & \\
\hline & & & $\%$ & 75.6 & 22.5 & 1.9 & & & \\
\hline & & \multirow[t]{2}{*}{ Total } & $\mathrm{F}$ & 227 & 68 & 4 & \multirow{2}{*}{2.75} & \multirow{2}{*}{0.466} & \\
\hline & & & $\%$ & 75.9 & 22.7 & 1.3 & & & \\
\hline \multirow[t]{6}{*}{1} & \multirow{6}{*}{$\begin{array}{l}\text { Activating the media } \\
\text { role in educating the } \\
\text { family to protect } \\
\text { children from } \\
\text { intellectual } \\
\text { delinquency }\end{array}$} & \multirow[t]{2}{*}{ Workers } & $\mathrm{F}$ & 75 & 15 & - & \multirow{2}{*}{2.83} & \multirow{2}{*}{0.375} & \multirow{6}{*}{2} \\
\hline & & & $\%$ & 83.3 & 16.7 & - & & & \\
\hline & & \multirow[t]{2}{*}{ Students } & $\mathrm{F}$ & 154 & 50 & 5 & \multirow{2}{*}{2.71} & \multirow{2}{*}{0.504} & \\
\hline & & & $\%$ & 73.7 & 23.9 & 2.4 & & & \\
\hline & & \multirow[t]{2}{*}{ Total } & $\mathrm{F}$ & 229 & 65 & 5 & \multirow{2}{*}{2.75} & \multirow{2}{*}{0.471} & \\
\hline & & & $\%$ & 76.6 & 21.7 & 1.7 & & & \\
\hline \multirow[t]{6}{*}{10} & & Workers & $\mathrm{F}$ & 68 & 22 & - & & & \\
\hline & children's fulfillment & & $\%$ & 75.6 & 24.4 & - & 2.70 & 0.432 & \\
\hline & & Students & $\mathrm{F}$ & 161 & 42 & 6 & & 0.500 & \\
\hline & & & $\%$ & 77.0 & 20.1 & 2.9 & 2.74 & 0.500 & 3 \\
\hline & & Total & $\mathrm{F}$ & 229 & 64 & 6 & & 0,480 & \\
\hline & & & $\%$ & 76.6 & 21.4 & 2.0 & 2.75 & 0.400 & \\
\hline 7 & Strengthening & Workers & $\mathrm{F}$ & 71 & 19 & - & & & \\
\hline & relationships between & & $\%$ & 78.9 & 21.1 & - & 2.79 & 0.410 & \\
\hline & parents and children & Students & $\mathrm{F}$ & 161 & 41 & 7 & & 0.512 & 4 \\
\hline & & & $\%$ & 77.0 & 19.6 & $3 \cdot 3$ & 2.74 & 0.512 & 4 \\
\hline & & Total & $\mathrm{F}$ & 232 & 60 & 7 & 2.75 & 0.484 & \\
\hline & & & $\%$ & 77.6 & 20.1 & 2.3 & 2.15 & 0.404 & \\
\hline
\end{tabular}




\begin{tabular}{|c|c|c|c|c|c|c|c|c|c|}
\hline \multirow[t]{2}{*}{ No. } & \multirow{2}{*}{\multicolumn{2}{|c|}{ Item }} & \multicolumn{4}{|c|}{ Agreement } & \multirow{2}{*}{$\begin{array}{c}\text { Arithmetic } \\
\text { mean }\end{array}$} & \multirow{2}{*}{$\begin{array}{l}\text { Standard } \\
\text { deviation }\end{array}$} & \multirow{2}{*}{ Rank } \\
\hline & & & & Agree & Undecided & Disagree & & & \\
\hline \multirow[t]{6}{*}{3} & \multirow{6}{*}{$\begin{array}{l}\text { Activating the role of } \\
\text { family institutions to } \\
\text { help resolve family } \\
\text { problems }\end{array}$} & \multirow[t]{2}{*}{ Workers } & $\mathrm{F}$ & 74 & 16 & - & \multirow{2}{*}{2.82} & \multirow{2}{*}{0.384} & \multirow{6}{*}{5} \\
\hline & & & $\%$ & 82.2 & 17.8 & - & & & \\
\hline & & \multirow[t]{2}{*}{ Students } & $\mathrm{F}$ & 151 & 54 & 4 & \multirow{2}{*}{2.70} & \multirow{2}{*}{0.498} & \\
\hline & & & $\%$ & 72.2 & 25.8 & 1.9 & & & \\
\hline & & \multirow[t]{2}{*}{ Total } & $\mathrm{F}$ & 225 & 70 & 4 & \multirow{2}{*}{2.74} & \multirow{2}{*}{0.469} & \\
\hline & & & $\%$ & $75 \cdot 3$ & 23.4 & 1.3 & & & \\
\hline \multirow[t]{6}{*}{4} & \multirow{6}{*}{$\begin{array}{l}\text { Educating parents } \\
\text { about appropriate } \\
\text { parenting styles }\end{array}$} & \multirow[t]{2}{*}{ Workers } & $\mathrm{F}$ & 71 & 19 & - & \multirow{2}{*}{2.79} & & \\
\hline & & & $\%$ & 78.9 & 21.1 & - & & 0.410 & \\
\hline & & Students & $\mathrm{F}$ & 157 & 46 & 6 & 272 & 0500 & 6 \\
\hline & & & $\%$ & 75.1 & 22.0 & 2.9 & 2.72 & 0.509 & 0 \\
\hline & & Total & $\mathrm{F}$ & 228 & 65 & 6 & & & \\
\hline & & & $\%$ & 76.3 & 21.7 & 2.0 & 2.74 & 0.482 & \\
\hline 9 & Balance in treating & Workers & $\mathrm{F}$ & 69 & 20 & 1 & & & \\
\hline & children & & $\%$ & 76.7 & 22.2 & 1.1 & 2.76 & 0.457 & \\
\hline & & Students & $\mathrm{F}$ & 152 & 56 & 1 & & & \\
\hline & & & $\%$ & 72.7 & 26.8 & 0.5 & 2.72 & 0.459 & 7 \\
\hline & & Total & $\mathrm{F}$ & 221 & 76 & 2 & & & \\
\hline & & & $\%$ & 73.9 & 25.4 & 0.7 & 2.73 & 0.458 & \\
\hline 2 & Educating the family & Workers & $\mathrm{F}$ & 70 & 19 & 1 & 277 & & \\
\hline & about the necessity of & & $\%$ & 77.8 & 21.1 & 1.1 & 2.77 & $0.45^{1}$ & \\
\hline & family dialog & Students & $\mathrm{F}$ & 154 & 51 & 4 & & & 8 \\
\hline & & & $\%$ & 73.7 & 24.4 & 1.9 & 2.72 & 0.492 & 8 \\
\hline & & Total & $\mathrm{F}$ & 224 & 70 & 5 & & 0.480 & \\
\hline & & & $\%$ & 74.9 & 23.4 & 1.7 & 2.73 & 0.480 & \\
\hline 6 & The vital role of & Workers & $\mathrm{F}$ & 69 & 20 & 1 & & & \\
\hline & parents in helping & & $\%$ & 76.7 & 22.2 & 1.1 & 2.76 & 0.457 & \\
\hline & students solve their & Students & $\mathrm{F}$ & 152 & 50 & 7 & & & \\
\hline & children's problems & & $\%$ & 72.7 & 23.9 & 3.3 & 2.69 & 0.630 & 9 \\
\hline & & Total & $\mathrm{F}$ & 221 & 70 & 8 & 2.7 & 0.509 & \\
\hline & & & $\%$ & 73.9 & 23.4 & 2.7 & 2.71 & 0.509 & \\
\hline 11 & Parents' interest in & Workers & $\mathrm{F}$ & 67 & 23 & - & & & \\
\hline & contemporary & & $\%$ & 74.4 & 25.6 & - & 2.74 & 0.439 & \\
\hline & knowledge and & Students & $\mathrm{F}$ & 144 & 59 & 6 & 2,66 & & \\
\hline & & & $\%$ & 68.9 & 28.2 & 2.9 & 2.66 & 0.532 & 10 \\
\hline & & Total & $\mathrm{F}$ & 211 & 82 & 6 & & & \\
\hline & & & $\%$ & 70.6 & $27 \cdot 4$ & 2.0 & 2.69 & 0.509 & \\
\hline 5 & Activating the family & Workers & $\mathrm{F}$ & 71 & 18 & 1 & & & \\
\hline & role in helping & & $\%$ & 78.9 & 20.0 & 1.1 & 2.78 & 0.444 & \\
\hline & children choose & Students & $\mathrm{F}$ & 123 & 77 & 9 & & & \\
\hline & friends & & $\%$ & 58.9 & 36.8 & $4 \cdot 3$ & 2.55 & 0.579 & 11 \\
\hline & & Total & $\mathrm{F}$ & 194 & 95 & 10 & & & \\
\hline & & & $\%$ & 64.9 & 31.8 & $3 \cdot 3$ & 2.62 & 0.552 & \\
\hline Ove & 11 mean & & & & & & & & 36 \\
\hline
\end{tabular}

Table (8) shows that the arithmetic means of the suggested ways of preventing the family factors of intellectual delinquencies among youth ranged (2.75:2.62). While (giving the opportunity within the family to exchange opinions and hear the other's opinion) was ranked first, (activating the family role in helping children choose friends) was ranked last. Social workers agreed that (activating the media role in educating the family to protect children from intellectual delinquency) was ranked first. The participant students agreed that (giving the opportunity within the family to exchange opinions and hear the other's opinion) was ranked first. Additionally, (family interest in children's fulfillment of religious tasks) was ranked first. 
Table 9: Responses of the participants to the suggested ways of preventing the societal factors of intellectual delinquencies among youth

\begin{tabular}{|c|c|c|c|c|c|c|c|c|c|}
\hline \multirow[t]{2}{*}{ No. } & \multirow{2}{*}{\multicolumn{2}{|c|}{ Item }} & \multicolumn{4}{|c|}{ Agreement } & \multirow{2}{*}{$\begin{array}{l}\text { Arithmetic } \\
\text { mean }\end{array}$} & \multirow[b]{2}{*}{$\begin{array}{l}\text { Standard } \\
\text { deviation }\end{array}$} & \multirow[b]{2}{*}{ Rank } \\
\hline & & & \multirow{3}{*}{\begin{tabular}{|c|}
$\begin{array}{c}\text { Percentage } \\
\%\end{array}$ \\
$\mathrm{~F}$ \\
$\%$ \\
\end{tabular}} & \multirow{2}{*}{\begin{tabular}{|c|} 
Agree \\
73 \\
\end{tabular}} & \multirow{2}{*}{\begin{tabular}{|c|} 
Undecided \\
17
\end{tabular}} & Disagree & & & \\
\hline \multirow[t]{6}{*}{2} & \multirow{6}{*}{$\begin{array}{l}\text { Opening several work fields to face } \\
\text { unemployment }\end{array}$} & \multirow[t]{2}{*}{ Workers } & & & & - & \multirow{2}{*}{2.81} & \multirow{2}{*}{0.394} & \multirow{6}{*}{1} \\
\hline & & & & 81.1 & 18.9 & - & & & \\
\hline & & Students & $\mathrm{F}$ & 168 & 39 & 2 & & 0128 & \\
\hline & & & $\%$ & 80.4 & 18.7 & 1.0 & 2.79 & 0.428 & \\
\hline & & Total & $\mathrm{F}$ & 241 & 56 & 2 & & & \\
\hline & & & $\%$ & 80.6 & 18.7 & 0.7 & 2.80 & 0.418 & \\
\hline 8 & Including youth needs in the national & Workers & $\mathrm{F}$ & 71 & 19 & - & & & \\
\hline & strategic plans and educating youth & & $\%$ & 78.9 & 21.1 & - & 2.79 & 0.410 & \\
\hline & about them & Students & $\mathrm{F}$ & 153 & 53 & 3 & & & \\
\hline & & & $\%$ & 73.2 & 25.4 & 1.4 & 2.72 & 0.482 & 2 \\
\hline & & Total & $\mathrm{F}$ & 224 & 72 & 3 & & & \\
\hline & & & $\%$ & 74.9 & 24.1 & 1.0 & 2.74 & 0.462 & \\
\hline 7 & Increasing the number if youth clubs and & Workers & $\mathrm{F}$ & 71 & 18 & 1 & 278 & & \\
\hline & entertainment institutions to spend & & $\%$ & 78.9 & 20.0 & 1.1 & 2.78 & 0.444 & \\
\hline & & Students & $\mathrm{F}$ & 156 & 49 & 4 & & & \\
\hline & & & $\%$ & 74.6 & 23.4 & 1.9 & 2.73 & 0.488 & 3 \\
\hline & & Total & $\mathrm{F}$ & 227 & 67 & 5 & & & \\
\hline & & & $\%$ & 75.9 & 22.4 & 1.7 & 2.74 & 0.475 & \\
\hline 6 & Activating the role of social institutions & Workers & $\mathrm{F}$ & 72 & 18 & - & & & \\
\hline & (charities/ social centers/ universities) in & & $\%$ & 80.0 & 20.0 & - & 2.80 & 0.402 & \\
\hline & & Students & $\mathrm{F}$ & 145 & 60 & 4 & & & \\
\hline & & & $\%$ & 69.4 & 28.7 & 1.9 & 2.67 & 0.509 & 4 \\
\hline & & Total & $\mathrm{F}$ & 217 & 78 & 4 & & & \\
\hline & & & $\%$ & 72.6 & 26.1 & 1.3 & 2.71 & 0.482 & \\
\hline 10 & Promoting the social responsibility & Workers & $\mathrm{F}$ & 71 & 18 & 1 & 78 & & \\
\hline & among youth & & $\%$ & 78.9 & 20.0 & 1.1 & 2.78 & 0.444 & \\
\hline & & Students & $\mathrm{F}$ & 145 & 58 & 6 & 267 & & \\
\hline & & & $\%$ & 69.4 & 27.8 & 2.9 & 2.67 & 0.531 & 5 \\
\hline & & Total & $\mathrm{F}$ & 216 & 76 & 7 & & & \\
\hline & & & $\%$ & 72.2 & 25.4 & 2.3 & 2.70 & 0.508 & \\
\hline 3 & Raising censorship of media, especially & Workers & $\mathrm{F}$ & 68 & 22 & - & 2.76 & 0.422 & \\
\hline & social media & & $\%$ & 75.6 & 24.4 & - & 2.76 & 0.432 & \\
\hline & & Students & $\mathrm{F}$ & 149 & 51 & 9 & & & \\
\hline & & & $\%$ & 71.3 & 24.4 & 4.3 & 2.67 & 0.556 & 6 \\
\hline & & Total & $\mathrm{F}$ & 217 & 73 & 9 & & & \\
\hline & & & $\%$ & 72.6 & 24.4 & 3.0 & 2.70 & 0.522 & \\
\hline 9 & Planning supporting programs to & Workers & $\mathrm{F}$ & 71 & 18 & 1 & -8 & 0 & \\
\hline & promote citizenship and belonging & & $\%$ & 78.9 & 20.0 & 1.1 & 2.78 & 0.444 & \\
\hline & among youth & Students & $\mathrm{F}$ & 144 & 56 & 9 & & & \\
\hline & & & $\%$ & 68.9 & 26.8 & 4.3 & 2.65 & 0.562 & 7 \\
\hline & & Total & $\mathrm{F}$ & 215 & 74 & 10 & 260 & 0522 & \\
\hline & & & $\%$ & 71.9 & 24.7 & $3 \cdot 3$ & 2.69 & 0.532 & \\
\hline 4 & Interest in the quick punishment of & Workers & $\mathrm{F}$ & 67 & 22 & 1 & & & \\
\hline & intellectual delinquents & & $\%$ & 74.4 & 24.4 & 1.1 & 2.73 & 0.469 & \\
\hline & & Students & $\mathrm{F}$ & 144 & 59 & 6 & 66 & & 8 \\
\hline & & & $\%$ & 68.9 & 28.2 & 2.9 & 2.66 & 0.532 & 8 \\
\hline & & Total & $\mathrm{F}$ & 211 & 81 & 7 & & & \\
\hline & & & $\%$ & 70.6 & 27.1 & 2.3 & 2.68 & 0.514 & \\
\hline 1 & Formulating clear and active systems of & Workers & $\mathrm{F}$ & 69 & 21 & - & & & \\
\hline & youth care & & $\%$ & 76.7 & 23.3 & - & 2.77 & 0.425 & \\
\hline & & Students & $\mathrm{F}$ & 143 & 58 & 8 & 26 & 0554 & \\
\hline & & & $\%$ & 68.4 & 27.8 & 3.8 & 2.05 & 0.554 & 9 \\
\hline & & Total & $\mathrm{F}$ & 212 & 79 & 8 & & & \\
\hline & & & $\%$ & 70.9 & 26.4 & 2.7 & 2.68 & 0.521 & \\
\hline
\end{tabular}




\begin{tabular}{|c|c|c|c|c|c|c|c|c|c|}
\hline No. & \multicolumn{2}{|l|}{ Item } & \multicolumn{4}{|c|}{ Agreement } & $\begin{array}{c}\text { Arithmetic } \\
\text { mean }\end{array}$ & $\begin{array}{l}\text { Standard } \\
\text { deviation }\end{array}$ & Rank \\
\hline \multirow{6}{*}{5} & \multirow{6}{*}{$\begin{array}{l}\text { Guiding mass media to adhere to the } \\
\text { societal policy in achieving intellectual } \\
\text { security }\end{array}$} & \multirow{2}{*}{ Workers } & $\mathrm{F}$ & 70 & 20 & - & \multirow{2}{*}{2.78} & \multirow{2}{*}{0.418} & \multirow{6}{*}{10} \\
\hline & & & $\%$ & 77.8 & 22.2 & - & & & \\
\hline & & \multirow[t]{2}{*}{ Students } & $\mathrm{F}$ & 135 & 68 & 6 & \multirow{2}{*}{2.62} & \multirow[b]{2}{*}{0.543} & \\
\hline & & & $\%$ & 64.6 & 32.5 & 2.9 & & & \\
\hline & & \multirow[t]{2}{*}{ Total } & $\mathrm{F}$ & 205 & 88 & 6 & \multirow{2}{*}{2.67} & \multirow{2}{*}{0.513} & \\
\hline & & & $\%$ & 68.6 & 29.4 & 2.0 & & & \\
\hline \multirow[t]{6}{*}{11} & \multirow{6}{*}{$\begin{array}{l}\text { Defining the image, characteristics, and } \\
\text { shapes of the enemy in the minds of the } \\
\text { youth and qualifying them to be good } \\
\text { citizens }\end{array}$} & \multirow[t]{2}{*}{ Workers } & $\mathrm{F}$ & 63 & 23 & 4 & \multirow{2}{*}{2.66} & \multirow{2}{*}{0.564} & \multirow{6}{*}{11} \\
\hline & & & $\%$ & 70.0 & 25.6 & 4.4 & & & \\
\hline & & \multirow[t]{2}{*}{ Students } & $\mathrm{F}$ & 141 & 64 & 4 & \multirow{2}{*}{2.66} & \multirow{2}{*}{0.515} & \\
\hline & & & $\%$ & 67.5 & 30.6 & 1.9 & & & \\
\hline & & \multirow[t]{2}{*}{ Total } & $\mathrm{F}$ & 204 & 87 & 8 & \multirow{2}{*}{2.66} & \multirow{2}{*}{0.529} & \\
\hline & & & $\%$ & 68.2 & 29.1 & 2.7 & & & \\
\hline \multicolumn{3}{|c|}{ Overall mean } & & & & & 2. & 71 & 150 \\
\hline
\end{tabular}

Table (9) shows that the arithmetic means of the suggested ways of preventing the societal factors of intellectual delinquencies among youth ranged (2.80:2.66). While (opening several work fields to face unemployment) was ranked first, (defining the image, characteristics, and shapes of the enemy in the minds of the youth and qualifying them to be good citizens) was ranked last.

Table 10: Responses of the participants to the suggested ways of preventing the personal factors of intellectual delinquencies among youth

\begin{tabular}{|c|c|c|c|c|c|c|c|c|c|}
\hline \multirow[t]{2}{*}{ No. } & \multirow{2}{*}{\multicolumn{2}{|c|}{ Item }} & \multicolumn{4}{|c|}{ Agreement } & \multirow{2}{*}{$\begin{array}{c}\text { Arithmetic } \\
\text { mean }\end{array}$} & \multirow{2}{*}{$\begin{array}{l}\text { Standard } \\
\text { deviation }\end{array}$} & \multirow{2}{*}{ Rank } \\
\hline & & & & Agree & Undecided & Disagree & & & \\
\hline \multirow[t]{6}{*}{6} & \multirow[t]{6}{*}{ Fostering stdents' self-confidence } & \multirow[t]{2}{*}{ Workers } & $\mathrm{F}$ & 71 & 18 & 1 & \multirow{2}{*}{2.78} & \multirow{2}{*}{0.444} & \multirow{6}{*}{1} \\
\hline & & & $\%$ & 78.9 & 20.0 & 1.1 & & & \\
\hline & & \multirow[t]{2}{*}{ Students } & $\mathrm{F}$ & 164 & 40 & 5 & \multirow{2}{*}{2.76} & \multirow[b]{2}{*}{0.481} & \\
\hline & & & $\%$ & 78.5 & 19.1 & 2.4 & & & \\
\hline & & \multirow[t]{2}{*}{ Total } & $\mathrm{F}$ & 235 & 58 & 6 & \multirow[b]{2}{*}{2.77} & \multirow{2}{*}{0.469} & \\
\hline & & & $\%$ & 78.6 & 19.4 & 0.2 & & & \\
\hline \multirow[t]{6}{*}{11} & \multirow{6}{*}{$\begin{array}{l}\text { Educating students to obtain knowledge } \\
\text { from sound resources }\end{array}$} & \multirow[t]{2}{*}{ Workers } & $\mathrm{F}$ & 69 & 21 & - & \multirow{2}{*}{2.77} & \multirow{2}{*}{0.425} & \multirow{6}{*}{2} \\
\hline & & & $\%$ & 76.7 & 23.3 & - & & & \\
\hline & & \multirow[t]{2}{*}{ Students } & $\mathrm{F}$ & 164 & 39 & 6 & \multirow{2}{*}{2.76} & & \\
\hline & & & $\%$ & 78.5 & 18.7 & 2.9 & & 0.493 & \\
\hline & & Total & $\mathrm{F}$ & 233 & 60 & 6 & 276 & 0.473 & \\
\hline & & & $\%$ & 77.9 & 20.1 & 0.2 & 2.70 & $0.4 / 3$ & \\
\hline 2 & Equipping & Workers & $\mathrm{F}$ & 70 & 20 & - & & 048 & \\
\hline & students with life skills & & $\%$ & 77.8 & 22.2 & - & 2.78 & 0.418 & \\
\hline & & Students & $\mathrm{F}$ & 157 & 49 & 3 & & & \\
\hline & & & $\%$ & 75.1 & 23.4 & 1.4 & 2.74 & 0.473 & 3 \\
\hline & & Total & $\mathrm{F}$ & 227 & 69 & 3 & & & \\
\hline & & & $\%$ & 75.9 & 23.1 & 1.0 & 2.75 & 0.457 & \\
\hline 3 & Helping students face their life & Workers & $\mathrm{F}$ & 69 & 21 & - & & & \\
\hline P & circumstances & & $\%$ & 76.7 & 23.3 & - & 2.77 & 0.425 & \\
\hline & & Students & $\mathrm{F}$ & 154 & 52 & 3 & & & \\
\hline & & & $\%$ & 73.7 & 24.9 & 1.4 & 2.72 & 0.480 & 4 \\
\hline & & Total & $\mathrm{F}$ & 223 & 73 & 3 & & 0464 & \\
\hline & & & $\%$ & 74.6 & 24.4 & 1.0 & 2.74 & 0.464 & \\
\hline 5 & Raising students' intellectual awareness & Workers & $\mathrm{F}$ & 71 & 18 & 1 & 2.78 & 0.444 & \\
\hline & & & $\%$ & 78.9 & 20.0 & 1.1 & 2.70 & 0.444 & \\
\hline & & Students & $\mathrm{F}$ & 154 & 52 & 3 & & 0480 & \\
\hline & & & $\%$ & 73.7 & 24.9 & 1.4 & 2.72 & 0.480 & 5 \\
\hline & & Total & $\mathrm{F}$ & 225 & 70 & 4 & & & \\
\hline & & & $\%$ & $75 \cdot 3$ & 23.4 & 1.3 & 2.74 & 0.469 & \\
\hline 8 & Developing students' citizenship & Workers & $\mathrm{F}$ & 73 & 16 & 1 & & & \\
\hline & & & $\%$ & 81.1 & 17.8 & 1.1 & 2.80 & 0.429 & \\
\hline & & Students & $\mathrm{F}$ & 152 & 52 & 5 & & & 6 \\
\hline & & & $\%$ & 72.7 & 24.9 & 2.4 & 2.70 & 0.508 & \\
\hline & & Total & $\mathrm{F}$ & 225 & 68 & 6 & 2.73 & 0.487 & \\
\hline
\end{tabular}




\begin{tabular}{|c|c|c|c|c|c|c|c|c|c|}
\hline \multirow[t]{3}{*}{ No. 1} & \multirow[t]{3}{*}{ Item } & \multirow[b]{3}{*}{$\mid$} & \multicolumn{4}{|c|}{ Agreement } & \multirow{3}{*}{$\begin{array}{c}\text { Arithmetic } \\
\text { mean }\end{array}$} & \multirow{3}{*}{$\begin{array}{l}\text { Standard } \\
\text { deviation }\end{array}$} & \multirow{3}{*}{ Rank } \\
\hline & & & \multicolumn{2}{|c|}{\begin{tabular}{|l|l|l|} 
Agree & Undecided & Disagree \\
\end{tabular}} & Undecided & & & & \\
\hline & & & $\%$ & $75 \cdot 3$ & 22.7 & \begin{tabular}{|l|}
0.2 \\
\end{tabular} & & & \\
\hline \multirow[t]{6}{*}{7} & \multirow{6}{*}{$\begin{array}{l}\text { Helping students engage in environment } \\
\text { service projects }\end{array}$} & \multirow[t]{2}{*}{ Workers } & $\mathrm{F}$ & 68 & 21 & 1 & \multirow{2}{*}{2.74} & \multirow{2}{*}{0.464} & \multirow{6}{*}{7} \\
\hline & & & $\%$ & 75.6 & 23.3 & 1.1 & & & \\
\hline & & \multirow[t]{2}{*}{ Students } & $\mathrm{F}$ & 152 & 52 & 5 & \multirow{2}{*}{2.70} & \multirow{2}{*}{0.508} & \\
\hline & & & $\%$ & 72.7 & 24.9 & 2.4 & & & \\
\hline & & \multirow[t]{2}{*}{ Total } & $\mathrm{F}$ & 220 & 73 & 6 & \multirow{2}{*}{2.72} & \multirow{2}{*}{0.494} & \\
\hline & & & $\%$ & 73.6 & 24.4 & 0.2 & & & \\
\hline \multirow[t]{6}{*}{9} & \multirow{6}{*}{$\begin{array}{l}\text { Equipping students with knowledge related } \\
\text { to intellectual security }\end{array}$} & \multirow{2}{*}{ Workers } & $\mathrm{F}$ & 70 & 19 & 1 & \multirow{2}{*}{2.77} & \multirow{2}{*}{0.451} & \\
\hline & & & $\%$ & 77.8 & 21.1 & 1.1 & & & \\
\hline & & \multirow[t]{2}{*}{ Students } & $\mathrm{F}$ & 147 & 59 & 3 & \multirow{2}{*}{2.69} & & \\
\hline & & & $\%$ & 70.3 & 28.2 & 1.4 & & 0.494 & 8 \\
\hline & & Total & $\mathrm{F}$ & 217 & 78 & 4 & & & \\
\hline & & & $\%$ & 72.6 & 26.1 & 1.3 & 2.71 & 0.482 & \\
\hline 1 & University role in students' self- & Workers & $\mathrm{F}$ & 69 & 20 & 1 & & & \\
\hline & development & & $\%$ & 76.7 & 22.2 & 1.1 & 2.76 & 0.457 & \\
\hline & & Students & $\mathrm{F}$ & 150 & 54 & 5 & 260 & 0.51 & 0 \\
\hline & & & $\%$ & 71.8 & 25.8 & 2.4 & 2.09 & 0.311 & 9 \\
\hline & & Total & $\mathrm{F}$ & 219 & 74 & 6 & 27 & 0406 & \\
\hline & & & $\%$ & 73.2 & 24.7 & 2.0 & $2 . / 1$ & 0.490 & \\
\hline 10 & Encouraging students to join the & Workers & $\mathrm{F}$ & 69 & 21 & - & & 0.425 & \\
\hline & extracurricular activities that support & & $\%$ & 76.7 & 23.3 & - & 2.77 & 0.425 & \\
\hline & intellectual security & Students & $\mathrm{F}$ & 147 & 51 & 11 & & 078 & \\
\hline & & & $\%$ & 70.3 & 24.4 & 5.3 & 2.65 & 0.578 & 10 \\
\hline & & Total & $\mathrm{F}$ & 216 & 72 & 11 & & & \\
\hline & & & $\%$ & 72.2 & 24.1 & $3 \cdot 7$ & 2.69 & 0.539 & \\
\hline 4 & Neuropsychological programming of & Workers & $\mathrm{F}$ & 60 & 26 & 4 & & & \\
\hline T & students to overcome extreme ideas that & & $\%$ & 66.7 & 28.9 & 4.4 & 2.62 & 0.572 & \\
\hline & hinder intellectual security & Students & $\mathrm{F}$ & 145 & 56 & 8 & & & \\
\hline & & & $\%$ & 69.4 & 26.8 & 3.8 & 2.66 & 0.551 & 11 \\
\hline & & Total & $\mathrm{F}$ & 205 & 82 & 12 & & & \\
\hline & & & $\%$ & 68.6 & 27.4 & 0.4 & 2.65 & 0.557 & \\
\hline Over & rall mean & & & & & & 2.72 & 0.447 & \\
\hline
\end{tabular}

Table (10) illustrates that the arithmetic means of the suggested ways of preventing the personal factors of intellectual delinquencies among youth ranged (2.77:2.65). While (fostering students' selfconfidence) was ranked first, (neuropsychological programming of students to overcome extreme ideas that hinder intellectual security) was ranked last. The social workers agreed that (developing students' citizenship) was ranked first. The participant students agreed that (fostering students' selfconfidence) and (educating students to obtain knowledge from sound resources) were ranked first.

Table 11: Responses of the participants to the suggested ways of preventing the educational factors of intellectual delinquencies among youth

\begin{tabular}{|c|c|c|c|c|c|c|c|c|c|}
\hline \multirow[t]{2}{*}{ No. } & \multirow{2}{*}{\multicolumn{2}{|c|}{ Item }} & \multicolumn{4}{|c|}{ Agreement } & \multirow{2}{*}{$\begin{array}{l}\text { Arithmetic } \\
\text { mean }\end{array}$} & \multirow{2}{*}{$\begin{array}{l}\text { Standard } \\
\text { deviation }\end{array}$} & \multirow{2}{*}{ Rank } \\
\hline & & & & Agree & Undecided & Disagree & & & \\
\hline \multirow[t]{6}{*}{11} & \multirow{6}{*}{$\begin{array}{l}\text { Sharing experience among (local and overseas) } \\
\text { universities on handling the issues of intellectual security }\end{array}$} & \multirow{2}{*}{ Workers } & $\mathrm{F}$ & 70 & 20 & - & \multirow{2}{*}{2.78} & \multirow{2}{*}{0.418} & \multirow{6}{*}{1} \\
\hline & & & $\%$ & 77.8 & 22.2 & - & & & \\
\hline & & \multirow{2}{*}{ Students } & $\mathrm{F}$ & 154 & 48 & 7 & \multirow{2}{*}{2.70} & \multirow{2}{*}{$0.5^{26}$} & \\
\hline & & & $\%$ & 73.7 & 23.0 & 3.3 & & & \\
\hline & & \multirow[t]{2}{*}{ Total } & $\mathrm{F}$ & 224 & 68 & 7 & \multirow{2}{*}{2.73} & \multirow{2}{*}{0.497} & \\
\hline & & & $\%$ & 74.9 & 22.7 & 2.3 & & & \\
\hline \multirow[t]{6}{*}{5} & \multirow{6}{*}{$\begin{array}{l}\text { Activating the role of social guidance departments to } \\
\text { help students solve their problems }\end{array}$} & \multirow[t]{2}{*}{ Workers } & $\mathrm{F}$ & 70 & 18 & 2 & \multirow{2}{*}{2.76} & \multirow{2}{*}{0.481} & \multirow{6}{*}{2} \\
\hline & & & $\%$ & 77.8 & 20.0 & 2.2 & & & \\
\hline & & \multirow[t]{2}{*}{ Students } & $\mathrm{F}$ & 153 & 50 & 6 & \multirow{2}{*}{2.70} & \multirow{2}{*}{0.517} & \\
\hline & & & $\%$ & 73.2 & 23.9 & 2.9 & & & \\
\hline & & \multirow[t]{2}{*}{ Total } & $\mathrm{F}$ & 223 & 68 & 8 & \multirow{2}{*}{2.72} & \multirow{2}{*}{0.506} & \\
\hline & & & $\%$ & 74.6 & 22.7 & 2.7 & & & \\
\hline \multirow[t]{5}{*}{3} & \multirow{5}{*}{$\begin{array}{l}\text { Interest in applying disciplinary regulations to } \\
\text { delinquents }\end{array}$} & \multirow[t]{2}{*}{ Workers } & $\mathrm{F}$ & 67 & 23 & - & \multirow{2}{*}{2.74} & \multirow{2}{*}{0.439} & \\
\hline & & & $\%$ & 74.4 & 25.6 & - & & & \\
\hline & & Students & $\mathrm{F}$ & 149 & 55 & 5 & 260 & 0.512 & 3 \\
\hline & & & $\%$ & 71.3 & 26.3 & 2.4 & 2.09 & $0.5^{13}$ & \\
\hline & & Total & $\mathrm{F}$ & 216 & 78 & 5 & 2.71 & 0.492 & \\
\hline
\end{tabular}




\begin{tabular}{|c|c|c|c|c|c|c|c|c|c|}
\hline \multirow[t]{3}{*}{ No. } & \multirow{3}{*}{ Item } & & \multicolumn{4}{|c|}{ Agreement } & \multirow{3}{*}{$\begin{array}{l}\text { Arithmetic } \\
\text { mean }\end{array}$} & \multirow{3}{*}{$\begin{array}{l}\text { Standard } \\
\text { deviation }\end{array}$} & \multirow{3}{*}{ Rank } \\
\hline & & & \multirow{2}{*}{\multicolumn{2}{|c|}{\begin{tabular}{|c|c|} 
& Agree \\
0 & 72.2 \\
\end{tabular}}} & \multirow{2}{*}{\begin{tabular}{|c|} 
Undecided \\
26.1
\end{tabular}} & \multirow{2}{*}{\begin{tabular}{|c|} 
Disagree \\
1.7
\end{tabular}} & & & \\
\hline & & & & & & & & & \\
\hline \multirow[t]{6}{*}{6} & \multirow[t]{6}{*}{ Interest in fulfilling the actual needs of students } & \multirow[t]{2}{*}{ Workers } & $\mathrm{F}$ & 69 & 20 & 1 & \multirow{2}{*}{2.76} & \multirow{2}{*}{0.457} & \multirow{6}{*}{4} \\
\hline & & & $\%$ & 76.7 & 22.2 & 1.1 & & & \\
\hline & & \multirow[t]{2}{*}{ Students } & $\mathrm{F}$ & 154 & 46 & 9 & \multirow{2}{*}{2.69} & \multirow{2}{*}{0.548} & \\
\hline & & & $\%$ & 73.7 & 22.0 & 4.3 & & & \\
\hline & & Total & $\mathrm{F}$ & 223 & 66 & 10 & & & \\
\hline & & & $\%$ & 74.6 & 22.1 & 3.3 & 2.71 & 0.522 & \\
\hline 9 & Coordination between university departments about & Workers & $\mathrm{F}$ & 70 & 18 & 2 & & & \\
\hline & planning to educate students intellectually & & $\%$ & 77.8 & 20.0 & 2.2 & 2.76 & 0.481 & \\
\hline & & Students & $\mathrm{F}$ & 145 & 56 & 8 & 266 & & \\
\hline & & & $\%$ & 69.4 & 26.8 & 3.8 & 2.66 & 0.551 & 5 \\
\hline & & Total & $\mathrm{F}$ & 215 & 74 & 10 & 60 & & \\
\hline & & & $\%$ & 71.9 & 24.7 & $3 \cdot 3$ & 2.69 & 0.532 & \\
\hline 2 & Support the faculty-student relationship for intellectual & Workers & $\mathrm{F}$ & 66 & 24 & - & 72 & & \\
\hline & communication to educate students about intellectual & & $\%$ & 73.3 & 26.7 & - & 2.73 & 0.445 & \\
\hline & & Students & $\mathrm{F}$ & 143 & 59 & 7 & 265 & 0.544 & 6 \\
\hline & & & $\%$ & 68.4 & 28.2 & 3.3 & 2.05 & 0.544 & 0 \\
\hline & & Total & $\mathrm{F}$ & 209 & 83 & 7 & 268 & & \\
\hline & & & $\%$ & 69.9 & 27.8 & 2.3 & 2.68 & 0.517 & \\
\hline 8 & Focusing on programs of using scientific knowledge in & Workers & $\mathrm{F}$ & 69 & 19 & 2 & & 0487 & \\
\hline & the service of intellectual security & & $\%$ & 76.7 & 21.1 & 2.2 & 2.74 & 0.487 & \\
\hline & & Students & $\mathrm{F}$ & 143 & 59 & 7 & 265 & & \\
\hline & & & $\%$ & 68.4 & 28.2 & 3.3 & 2.65 & 0.544 & 7 \\
\hline & & Total & $\mathrm{F}$ & 212 & 78 & 9 & 68 & $0=28$ & \\
\hline & & & $\%$ & 70.9 & 26.1 & 3.0 & 2.68 & 0.528 & \\
\hline 4 & Reconsidering the student activity plans and including & Workers & $\mathrm{F}$ & 68 & 21 & 1 & & 0464 & \\
\hline & intellectual awareness activities & & $\%$ & 75.6 & 23.3 & 1.1 & 2.74 & 0.464 & \\
\hline & & Students & $\mathrm{F}$ & 144 & 55 & 10 & 260 & & 8 \\
\hline & & & $\%$ & 68.9 & 26.3 & 4.8 & 2.64 & 0.572 & 8 \\
\hline & & Total & $\mathrm{F}$ & 212 & 76 & 11 & 267 & 0.543 & \\
\hline & & & $\%$ & 70.9 & 25.4 & 3.7 & 2.07 & 0.543 & \\
\hline 10 & Using university's social responsibility programs to & Workers & $\mathrm{F}$ & 70 & 19 & 1 & 277 & (151 & \\
\hline & develop student citizenship & & $\%$ & 77.8 & 21.1 & 1.1 & 2.77 & $0.45^{1}$ & \\
\hline & & Students & $\mathrm{F}$ & 135 & 67 & 7 & 26 & & \\
\hline & & & $\%$ & 64.6 & 32.1 & $3 \cdot 3$ & 2.61 & 0.553 & 9 \\
\hline & & Total & $\mathrm{F}$ & 205 & 86 & 8 & -66 & - & \\
\hline & & & $\%$ & 68.6 & 28.8 & 2.7 & 2.66 & 0.528 & \\
\hline 7 & Activating the role of academic coiunseling in guiding & Workers & $\mathrm{F}$ & 66 & 18 & 6 & $6-1$ & 60 & \\
\hline & students intellectually & & $\%$ & 73.3 & 20.0 & 6.7 & 2.67 & 0.600 & \\
\hline & & Students & $\mathrm{F}$ & 145 & 55 & 9 & $26=$ & -6 & 10 \\
\hline & & & $\%$ & 69.4 & 26.3 & 4.3 & 2.65 & 0.561 & 10 \\
\hline & & Total & $\mathrm{F}$ & 211 & 73 & 15 & 2.66 & 0.572 & \\
\hline & & & $\%$ & 70.6 & 24.4 & 5.0 & & $0.5 / 2$ & \\
\hline 1 & Linking courses to intellectual security mechanisms & Workers & $\mathrm{F}$ & 67 & 19 & 4 & 70 & 0.550 & \\
\hline & & & $\%$ & 74.4 & 21.1 & 4.4 & 2.70 & 0.550 & \\
\hline & & Students & $\mathrm{F}$ & 135 & 63 & 11 & & & 1 \\
\hline & & & $\%$ & 64.6 & 30.1 & 5.3 & 2.59 & 0.590 & 11 \\
\hline & & Total & $\mathrm{F}$ & 202 & 82 & 15 & & & \\
\hline & & & $\%$ & 67.6 & 27.4 & 5.0 & 2.63 & 0.579 & \\
\hline Ove & rall mean & & & & & & 2.68 & 0.477 & \\
\hline
\end{tabular}

Table (11) illustrates that the arithmetic means of the suggested ways of prevention ranged (2.73:2.63). While (sharing experience among (local and overseas) universities on handling the issues of intellectual security) was ranked first, (linking courses to intellectual security mechanisms) was ranked last.

\section{Discussion}

According to responses of the participants, a key obtained result to the intellectual delinquencies among youth is that (fanaticism) is ranked first whereas (calling for and promoting anti-national groups) is ranked last. The author argues that agreement on fanaticism as the highest intellectual 
delinquency is correlated with intellectual delinquency. Fanaticism and intellectual delinquency are two faces of the same coin; fanaticism is the cultural and intellectual face, and intellectual delinquency is the behavioral and social one. The author believes that this finding matches the characteristics of university students who tend to overcome rules to express themselves. Moreover, youth with intellectual delinquency tend to accept all aspects that promote their ideas, especially those that make them unique. This finding agrees with Al-tayyar (Al-tayyar 2017) that fanaticism, refusal of others' opinions, and aggression are manifestations of intellectual delinquency. The highlighted manifestations match functionalism's inputs that disorders in university students' personalities cause a state of conflict with society due to intellectual delinquency.

Concerning the family factors of intellectual delinquency among youth, the author argues that the lack of time dedicated to the behavior, monitoring, guiding, and solving the problems of children makes them easily prone to many dangerous family and social issues, such as intellectual delinquency. Because parents are preoccupied with securing children's future needs, they cannot monitor their conditions, resulting in family breakdown and intellectual delinquencies. The youth agreed that (lack of family dialog) was ranked first because parents do not dedicate time to speak to their children, making the latter fulfill their needs on their own, especially in adolescence and youth.

From the author's perspective regarding societal factors of intellectual delinquency among youth, the religious institutions are limited to disseminating the principles of Islam among students. The educational institution is interested in teaching students fruitful science. In contrast, the cultural institution is interested in developing and fostering art talents. Additionally, the lack of shared planning between these institutions to provide an intellectually secured environment to students plays a role. Moreover, legal punishment is the only method to prevent some youth from intellectual delinquencies due to the lack of knowledge.

With concern to responses of the participants to the personal factors of intellectual delinquency among youth, the author argues that the most harmful aspect of human personality is weak personality, lack of confidence, and low self-esteem, making it easy to be intellectually affected and have intellectual delinquency. A weak personality seeks to achieve the satisfaction of others without knowledge. The author argues that accepting unwanted actions makes the youth copy others. Thus, youth become easily dependent, driven by fear of others, low esteem, or weak personality.

As for the obtained result according to educational factors of intellectual delinquency among youth, the author argues that social guidance and counseling seeks to meet youth's basic needs, modify wrong ideas and beliefs, prevent delinquency, and solve problems individually and socially. Therefore, the weak role of these departments causes intellectual delinquency. The author argues that these differences cause different ideas and beliefs and promote fanaticism, threatening social texture and intellectual and cultural identity and causing intellectual delinquency. In sum, the author attributes the result of this question to various factors of intellectual delinquency among youth. This result agrees with the findings of Ronald (Ronald 2005; Alhokeel 2013; Al-Khataibeh et al. 2014; Alaly and Khair-Bek 2017).

With regard to the suggested ways of preventing the family factors of intellectual delinquencies among youth, the author argues that protecting youth from intellectual delinquency is achieved by promoting student awareness and developing their abilities to confront the cultural challenges of globalization, especially intellectual delinquency in the Saudi community. All types of media can play this role by educating families about the dangers of these delinquencies. The author argues that family plays a critical role in confronting intellectual delinquencies by exchanging opinions and ideas and accepting others' opinions. Furthermore, family is the basis of protecting children from deviant thinking by promoting religious awareness.

As for the result concerning the suggested ways of preventing the societal factors of intellectual delinquencies among youth, the author argues that unemployment causes many problems intellectually and socially. Thus, intellectual delinquencies may be prevented by employment.

Based on the result related to the suggested ways of preventing the personal factors of intellectual delinquencies among youth, the author argues that intellectual delinquencies negatively 
affect citizenship values, such as belonging, appreciating national interests, and respecting national leaders and institutions. Knowledge and awareness are important to overcome delinquency. The author argues that these items are highly important because they help overcome weak personalities and get fruitful knowledge to prevent misconceptions.

According to the obtained results concerning the suggested ways of preventing the educational factors of intellectual delinquencies among youth, the author argues that confronting intellectual delinquency requires the cooperation of all stakeholders, especially specialists and experts. Utilizing various mechanisms helps prevent intellectual delinquency ultimately. Moreover, a well-defined strategic planning process by adopting the author's proposal can help protect university students from intellectual delinquencies.

This finding agrees with the results of Dillon et al. (Dillon et al. 2008), Clinch (Clinch 2011), Alhokeel (Alhokeel 2013), and Alamry (Alamry 2014) that provide methods and mechanisms and make recommendations to protect youth from intellectual delinquencies.

\section{Recommendations}

The author suggests ways of preventing intellectual delinquency, such as

1. Establishing intellectual institutions and centers that foster intellectual security in society, disseminate intellectual awareness, and take care of and resolve youth's social and economic problems.

2. Establishing special units at the university to provide training courses to students to develop their skills and raise their awareness about current issues, such as intellectual delinquencies.

3. Including courses or syllabi to teach university students the preventive concepts towards intellectual delinquencies.

4. Providing a safer university environment in which university students practice their talents, develop skills, and act positively away from the manifestations of intellectual delinquency.

5. Activating the education role of societal institutions towards raising youth and protecting their ideas because of their guidance and counseling roles, as well as educational, cultural, and social programs and events.

6. Holding training workshops in family counseling centers to raise their awareness of intellectual delinquency and train them in the treatment means and professional skills, especially among youth.

7. Urging universities to hold conferences and symposia on the issues of intellectual delinquency to have constructive discussions with the youth to express opinions positively towards life issues.

8. Monitoring university students periodically to identify intellectual delinquencies, such as the tendency to isolation and aggression.

\section{Conclusion}

Intellectual delinquency is a complex phenomenon with several and overlapping causes. Its grave dangers affect both the person, the surrounding people, and the societal. Protecting the intellect of the societal members is a shared responsibility by the individual, family, and social institutions. Therefore, preventive means and intellectual treatments should be adopted.

\section{References}

Abdelgawad, M. (2009). Contemporary sociology theory (in Arabic). Amman: Dar Almaserra.

Abu-Arrad, S. (2010). University role in achieving intellectual security- A proposal (in Arabic). Arab Journal of Security Studies 27: 223-264. 
Al-Khataibeh, Y., Salameh, M. T. B., \& AL-Rawashdeh, A. Z. (2014). Factors and Manifestations of Ideological Extremism as Seen by Youth in Jordanian Universities: An Empirical Sociological Study. Journal of the Social Sciences 42: 9-45.

Al-tayyar, F. (2017). The role of high school in promoting intellectual awareness to prevent intellectual extremism (in Arabic). Al-Azhar Journal 173: 153-208.

Alaly, A, and G Khair-Bek. (2017). The impact of intellectual delinquency on social security (A Field study in Tishreen University) (in Arabic). Tishreen University Journal for Research and Scientific Studies 7: 763-777.

Alamry, A. (2014). The role of security culture in the prevention of extreme thought in the Saudi society (in Arabic). Naif Arab University for Security Sciences.

Aldahmash, K. (2019). Saudi efforts in confronting intellectual terrorism through educational institutions based on the new media: Social media (in Arabic). Journal of Mansoura Faculty of Education 107: 252-274.

Alhokeel, A. (2013). The role of academic institutions' directors at Imam Mohammad Ibn Saud Islamic University in preventing intellectual delinquency among students (in Arabic). IMSIU, Saudi Arabia: Imam Mohammad Ibn Saud Islamic University.

Aljahny, A. (2011). The role of education in protecting society from intellectual delinquency (in Arabic). Journal of Studies and Research 4: 248-277.

Alkhathlan, Z. (2017). A proposal for employing electronic communication tools in achieving intellectual security among youth in higher education institutions (in Arabic). International Interdisciplinary Journal of Education 6: 8-86.

Alkhatib, M. (2015). Intellectual delinquency and its relation to national security (in Arabic). Riyadh: Dar Ibn Khuzimah.

Alnaeemy, S. (2016). Security efforts in confronting intellectual delinquency (in Arabic). In Proceedings of Intellectual Delinquency Conference. Police Science Academy, Sharjah.

Alsharary, M. (2007). Attitudes of Saudi youth to terrorism: A study on a sample of the students of King Abdulaziz University in Jeddah (in Arabic)No Title. The University of Jordan.

Call, C. M. (2004). Intellectual safety and epistemological position in the college classroom. Ann Arbor: Cornell University.

Clinch, A. (2011). A community psychology approach to preventing violent extremism. Gaining the views of young people to inform primary prevention in secondary schools. England: University of Birmingham.

Dillon, F. R., Pantin, H., Robbins, M. S., \& Szapocznik, J. (2008). Exploring the Role of Parental Monitoring of Peers on the Relationship Between Family Functioning and Delinquency in the Lives of African American and Hispanic Adolescents. Crime $\mathcal{E}$ Delinquency 54. SAGE Publications Inc: 65-94. https://doi.org/10.1177/oo11128707305744.

Larton, P. (2005). Youth and problem of change. New York: Osaka Publisher.

Naser, G. (2007). Security media and awareness of deviation.

Ronald, W. (2005). Rational extremism: The calculus of discontent. Ontario: Ontario University of the Western Ontario.

Sonbol, M. (2013). Intellectual corruption among youth and the role of the service of the individual (in Arabic). Journal of Security Sciences and Training 58: 133-197. 